\title{
Two-dimensional correlation spectroscopy in polymer study
}

\author{
Yeonju Park ${ }^{1}$, Isao Noda ${ }^{2}$ and Young Mee Jung ${ }^{1 *}$ \\ ${ }^{1}$ Department of Chemistry, Kangwon National University, Chunchon, South Korea, ${ }^{2}$ Department of Materials Science and \\ Engineering, University of Delaware, Newark, DE, USA
}

This review outlines the recent works of two-dimensional correlation spectroscopy (2DCOS) in polymer study. 2DCOS is a powerful technique applicable to the in-depth analysis of various spectral data of polymers obtained under some type of perturbation. The powerful utility of 2DCOS combined with various analytical techniques in polymer studies and noteworthy developments of 2DCOS used in this field are also highlighted.

Keywords: two-dimensional correlation spectroscopy, 2DCOS, polymer, hetero-spectral correlation, projection 2D, PCA 2DCOS, eigenvalue manipulating transformation, self-modeling curve resolution

\section{OPEN ACCESS}

Edited by: Shigeaki Morita

Osaka Electro-Communication University, Japan

Reviewed by:

Michele Galizia,

University of Texas at Austin, USA Yusuke Hattori,

Musashino University, Japan

*Correspondence:

Young Mee Jung,

Department of Chemistry,

Kangwon National University,

1 Kangwondaehak-gil,

Chunchon 200-701, South Korea

ymjung@kangwon.ac.kr

Specialty section:

This article was submitted to Polymer Chemistry, a section of the journal

Frontiers in Chemistry

Received: 27 December 2014

Accepted: 17 February 2015

Published: 11 March 2015

Citation:

Park Y, Noda I and Jung YM (2015)

Two-dimensional correlation spectroscopy in polymer study. Front. Chem. 3:14.

doi: 10.3389/fchem.2015.00014

\section{Introduction}

Noda developed two-dimensional correlation spectroscopy (2DCOS) for analyzing the smallamplitude dynamic strain-dependent time-resolved IR linear dichroism spectra of a polymer film (Noda, 1986). Since the concept of 2DCOS was expanded to the various spectroscopic applications in 1993 (Noda, 1993), the generalized 2DCOS has become a very powerful analytical technique in many fields of spectroscopic studies, especially in polymer study.

The generalized 2DCOS can elucidate information in spectral variations, e.g., IR (Kim et al., 2006b; Cerdà-Costa et al., 2009; Huang et al., 2009; Unger et al., 2009, 2011; Del Río et al., 2010; Huang and Kuo, 2010; Jelèić et al., 2010; Jia et al., 2010; Lee et al., 2010, 2012; Peng et al., 2010; Popescu and Vasile, 2010, 2011; Zheng et al., 2010; Cheng et al., 2011; Jin et al., 2011; Kuo and Liu, 2011; Musto et al., 2011; Quaroni et al., 2011; Wang and Wu, 2011; Zhang et al., 2011; Ando et al., 2012; Qu et al., 2012; Su et al., 2012; Wu et al., 2012; Chai et al., 2013; Lai and Wu, 2013; Park et al., 2013; Shinzawa et al., 2013; Wang et al., 2013, 2014; Galizia et al., 2014; Hou et al., 2014; Noda, 2014c; Seo et al., 2014), Raman (Radice et al., 2010; Tang et al., 2010; Ma et al., 2011; Ji et al., 2012; Pazderka and Kopecký Jr, 2012; Brewster et al., 2013; Grzeszczuk et al., 2013; Noda, 2014d), terahertz (THz) (Hoshina et al., 2012, 2014), X-ray (Guo et al., 2011), UVVis (Hong et al., 2005; Jiang and Wu, 2008; Sikirzhytski et al., 2012; Zhong et al., 2012), NMR (Oh et al., 2009; Li et al., 2013), fluorescence (Hur et al., 2011; Zhang et al., 2013), and even chromatography (Izawa et al., 2001), under various external perturbations, such as thermal, electrical, optical, magnetic, and chemical perturbations (Noda, 1986, 1993; Hong et al., 2005; Kim et al., 2006b; Jiang and Wu, 2008; Cerdà-Costa et al., 2009; Huang et al., 2009; Oh et al., 2009; Unger et al., 2009; Del Río et al., 2010; Huang and Kuo, 2010; Jelèić et al., 2010; Jia et al., 2010; Lee et al., 2010, 2012; Peng et al., 2010; Popescu and Vasile, 2010, 2011; Radice et al., 2010; Tang et al., 2010; Zheng et al., 2010; Zhang et al., 2011; Cheng et al., 2011; Guo et al., 2011; Jin et al., 2011; Kuo and Liu, 2011; Ma et al., 2011; Quaroni et al., 2011; Unger et al., 2011; Wang and Wu, 2011; Ando et al., 2012; Hoshina et al., 2012, 2014; Ji et al., 2012; Pazderka and Kopecký Jr, 2012; Qu et al., 2012; Sikirzhytski et al., 2012; Su et al., 2012; Wu et al., 2012; Zhong et al., 2012; Brewster et al., 2013; Chai et al., 2013; Grzeszczuk et al., 2013; Lai and Wu, 2013; Li et al., 2013; Park et al., 2013; Shinzawa et al., 2013; Wang et al., 2013, 2014; Hou et al., 2014; Noda, 2014c,d; Seo et al., 2014). IR spectroscopy is the most common analytical probes used in 2DCOS 
(Kim et al., 2006b; Cerdà-Costa et al., 2009; Huang et al., 2009; Unger et al., 2009, 2011; Del Río et al., 2010; Huang and Kuo, 2010; Jelèić et al., 2010; Jia et al., 2010; Lee et al., 2010, 2012; Peng et al., 2010; Popescu and Vasile, 2010, 2011; Zheng et al., 2010; Cheng et al., 2011; Jin et al., 2011; Kuo and Liu, 2011; Musto et al., 2011; Quaroni et al., 2011; Wang and $\mathrm{Wu}, 2011$; Zhang et al., 2011; Ando et al., 2012; Qu et al., 2012; Su et al., 2012; Wu et al., 2012; Chai et al., 2013; Lai and Wu, 2013; Park et al., 2013; Shinzawa et al., 2013; Wang et al., 2013, 2014; Galizia et al., 2014; Hou et al., 2014; Noda, 2014c; Seo et al., 2014). The most popularly applied external perturbation in 2DCOS is temperature (Kim et al., 2006b; Unger et al., 2009, 2011; Jia et al., 2010; Peng et al., 2010; Popescu and Vasile, 2010; Tang et al., 2010; Zheng et al., 2010; Cheng et al., 2011; Wang and Wu, 2011; Pazderka and Kopecký Jr, 2012; Chai et al., 2013; Li et al., 2013; Wang et al., 2013, 2014; Hoshina et al., 2014; Hou et al., 2014; Seo et al., 2014). Applications of $2 \mathrm{DCOS}$ in investigations of intriguing properties of polymer system measured by different types of analytical probes has been substantially increased (Izawa et al., 2001; Hong et al., 2005; Kim et al., 2006b; Jiang and Wu, 2008; Huang et al., 2009; Oh et al., 2009; Unger et al., 2009, 2011; Del Río et al., 2010; Huang and Kuo, 2010; Jelèić et al., 2010; Jia et al., 2010; Lee et al., 2010, 2012; Peng et al., 2010; Popescu and Vasile, 2010, 2011; Radice et al., 2010; Tang et al., 2010; Zheng et al., 2010; Cheng et al., 2011; Guo et al., 2011; Jin et al., 2011; Kuo and Liu, 2011; Ma et al., 2011; Wang and Wu, 2011; Zhang et al., 2011; Ando et al., 2012; Hoshina et al., 2012, 2014; Qu et al., 2012; Su et al., 2012; Wu et al., 2012; Zhong et al., 2012; Chai et al., 2013; Grzeszczuk et al., 2013; Lai and Wu, 2013; Park et al., 2013; Shinzawa et al., 2013; Wang et al., 2013, 2014; Hou et al., 2014; Noda, 2014c,d; Seo et al., 2014). Generalized 2D correlation spectra has notable advantages: examination of inter- or intra-molecular interactions and determination of the sequential order of events, which is hardly depicted in conventional spectroscopy.

In this review, the background of the generalized 2DCOS is briefly discussed, and the powerful applications of 2DCOS in the studies of polymers are presented. Illustrative examples of 2DOCS in polymer research describing the improved information gained with noteworthy developments of 2DCOS are also provided.

\section{Background}

\section{Generalized 2D Correlation Spectroscopy}

The detailed background of the generalized 2DCOS is well introduced in books and book chapters (Noda, 2002, 2009; Ozaki, 2002; Noda and Ozaki, 2004; Ozaki and Šašic, 2005; Ozaki and Noda, 2006; Noda and Lindsey, 2010; Czarnik-Matusewicz and Jung, 2014; Jung and Noda, 2014) and review articles (Noda et al., 1993, 2000; Noda, 2000, 2004, 2006, 2007, 2008, 2014a,b; Jung and Noda, 2014). Here, we briefly describe the basic concept of 2DCOS.

In 2DCOS, a set of spectra $A\left(v_{j}, t_{i}\right)$ is obtained as a function of the spectral variable $v_{\mathrm{j}}$ with $j=1,2, \ldots n$ and some perturbation variable $t_{i}$ with $i=1,2, \ldots m$ during a well-defined observation interval between $t_{1}$ and $t_{m}$. A series of perturbationinduced dynamic spectra collected in a systematic manner are transformed into a set of $2 \mathrm{D}$ correlation spectra by a simple cross correlation analysis as shown in Figure 1.

The dynamic spectrum $\tilde{A}\left(v_{j}, t_{i}\right)$ of a system induced by the application of an external perturbation is defined formally within the observation interval between $t_{1}$ and $t_{m}$ as

$$
\tilde{A}\left(v_{j}, t_{i}\right)=A\left(v_{j}, t_{i}\right)-\bar{A}\left(v_{j}\right)
$$

where $\bar{A}\left(v_{j}\right)$ is the reference spectrum of the system. The reference spectrum is mostly selected as the stationary or averaged spectrum given by

$$
\bar{A}\left(v_{j}\right)=\frac{1}{m} \sum_{i=1}^{m} A\left(v_{j}, t_{i}\right)
$$

Synchronous $\Phi\left(v_{1}, v_{2}\right)$ and asynchronous $\Psi\left(v_{1}, v_{2}\right)$ correlation spectra are given by

$$
\begin{gathered}
\Phi\left(v_{1}, v_{2}\right)=\frac{1}{m-1} \sum_{i=1}^{m} \tilde{A}\left(v_{1}, t_{i}\right) \cdot \tilde{A}\left(v_{2}, t_{i}\right) \\
\Psi\left(v_{1}, v_{2}\right)=\frac{1}{m-1} \sum_{i=1}^{m} \tilde{A}\left(v_{1}, t_{i}\right) \cdot \sum_{k=1}^{m} N_{i k} \tilde{A}\left(v_{2}, t_{k}\right)
\end{gathered}
$$

where, $N_{i k}$ is the elements of so-called Hilbert-Noda transformation matrix given by

$$
N_{i k}=\left\{\begin{array}{cc}
0 & \text { if } i=k \\
\frac{1}{\pi(k-i)} & \text { otherwise }
\end{array}\right.
$$

\section{D Correlation Spectra}

The simultaneous or coincidental changes of spectral intensities at $v_{1}$ and $v_{2}$ are represented in the synchronous 2D correlation spectrum. Positive correlation peaks on the diagonal in synchronous $2 \mathrm{D}$ correlation spectrum correspond to the autocorrelation functions of spectral intensity variations, which are called autopeaks. Cross peaks, which are located at off-diagonal in synchronous 2D correlation spectrum, represent the coincidental or simultaneous changes of spectral intensities observed at two different spectral variables. The positive cross peaks depict that the intensities at corresponding spectral variables increase or decrease together. On the other hand, the negative cross peaks depict that one of the spectral intensities is increasing while the other is decreasing.

In contrast, the sequential, or unsynchronized, changes of spectral intensities at $v_{1}$ and $v_{2}$ are represented in the asynchronous $2 \mathrm{D}$ correlation spectrum. Asynchronous 2D correlation spectrum consists of only cross peaks, which shows an antisymmetric cross peaks with respect to the main diagonal. From the sign of cross peaks in $2 \mathrm{D}$ correlation spectra, the sequential changes in spectral intensities observed under the external perturbation can be determined. The same signs of synchronous and asynchronous cross peaks represent that the intensity change at $v_{1}$ occurs before $v_{2}$. While the different signs of synchronous and asynchronous cross peaks represent that the intensity change at $v_{2}$ occurs before $v_{1}$. This rule to determine sequential order of intensity variations is named Noda's rule. 


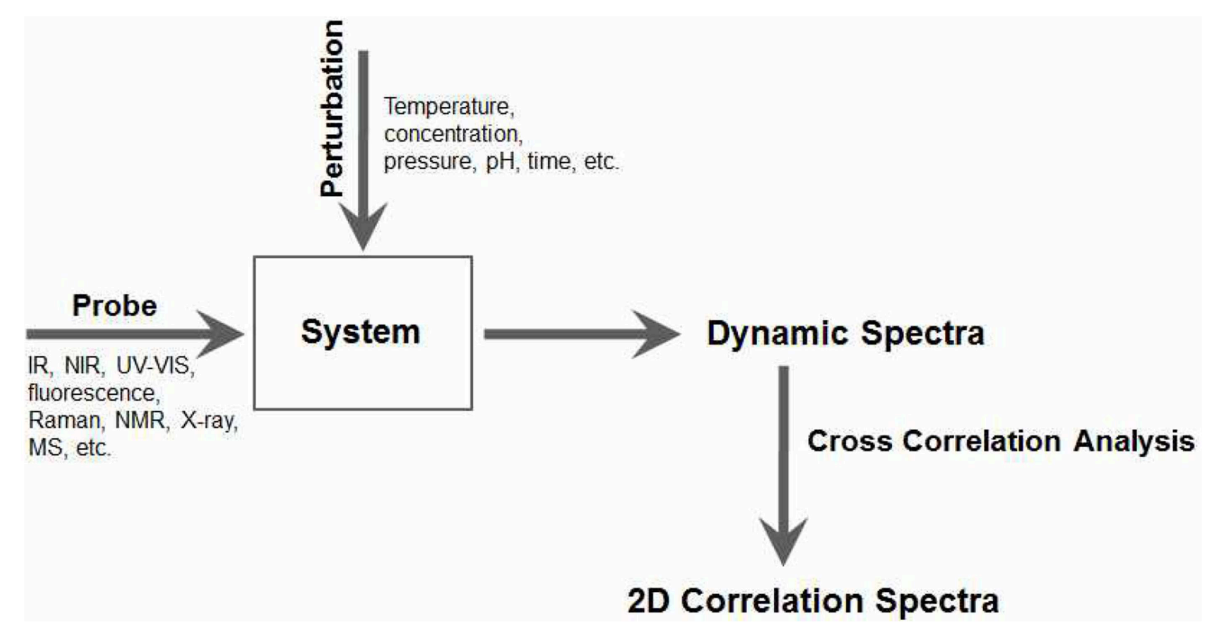

FIGURE 1 | The general scheme for constructing generalized 2D correlation spectra.

\section{Application of 2DCOS in Polymer Studies}

2DCOS, which can provide the easier access to the pertinent information in characterizing polymers, has been broadly applied to polymer studies to obtain new insights at the molecular level into the understanding behavior of polymers under an influence of an external perturbation. Various polymer systems, such as block copolymers (Kim et al., 2006b; Jia et al., 2010; Jin et al., 2011), biodegradable polymers (Guo et al., 2011; Unger et al., 2011; Ando et al., 2012; Hoshina et al., 2012, 2014; Wang et al., 2014), conducting polymer (Hong et al., 2005; Grzeszczuk et al., 2013), liquid crystals (Tang et al., 2010; Cheng et al., 2011), polymer blends (Oh et al., 2009; Unger et al., 2009, 2011; Jelèić et al., 2010; Popescu and Vasile, 2010, 2011; Kuo and Liu, 2011), and polymer nanocomposites (Huang et al., 2009; Huang and Kuo, 2010; Peng et al., 2010; Ando et al., 2012; Qu et al., 2012), etc., are analyzed by $2 \mathrm{DCOS}$. Detailed information of polymers for polymerization (Izawa et al., 2001; Hong et al., 2005; Huang and Kuo, 2010; Qu et al., 2012; Noda, 2014d; Seo et al., 2014), melting behavior (Unger et al., 2009; Peng et al., 2010; Popescu and Vasile, 2010), crystallization (Huang et al., 2009; Zheng et al., 2010; Guo et al., 2011; Unger et al., 2011; Ando et al., 2012; Hoshina et al., 2012, 2014; Chai et al., 2013; Wang et al., 2013, 2014),gelation (Wang and Wu, 2011; Su et al., 2012; Park et al., 2013), photoreaction (Lee et al., 2010, 2012), hydration (Lai and Wu, 2013), sorption/desorption processes (Musto et al., 2011; Lai and Wu, 2013; Galizia et al., 2014), and phase transition/separation (Kim et al., 2006b; Cheng et al., 2011; Kuo and Liu, 2011; Hou et al., 2014), etc., which are undergoing under the influence of applied external perturbation, are obtained by 2DCOS. 2DCOS probes with various analytical techniques, such as IR, near-IR (NIR), Raman, $\mathrm{X}$-ray, UV-Vis, THz, NMR spectroscopies, and even chromatography, have been successfully applied in polymer studies. 2DCOS has been extensively used in the IR study of polymers (Kim et al., 2006b; Huang et al., 2009; Unger et al., 2009, 2011; Del Río et al., 2010; Huang and Kuo, 2010; Jelèić et al., 2010; Jia et al., 2010; Lee et al., 2010, 2012; Peng et al., 2010; Popescu and Vasile, 2010,
2011; Zheng et al., 2010; Cheng et al., 2011; Jin et al., 2011; Kuo and Liu, 2011; Musto et al., 2011; Wang and Wu, 2011; Zhang et al., 2011; Ando et al., 2012; Qu et al., 2012; Su et al., 2012; Wu et al., 2012; Chai et al., 2013; Lai and Wu, 2013; Park et al., 2013; Shinzawa et al., 2013; Wang et al., 2013, 2014; Galizia et al., 2014; Hou et al., 2014; Noda, 2014c; Seo et al., 2014). Among the applications of 2DCOS in polymer study, temperature is the most used as an applied external perturbation (Kim et al., 2006b; Unger et al., 2009, 2011; Jia et al., 2010; Peng et al., 2010; Popescu and Vasile, 2010; Tang et al., 2010; Zheng et al., 2010; Cheng et al., 2011; Wang and Wu, 2011; Su et al., 2012; Wang et al., 2013, 2014; Hoshina et al., 2014; Hou et al., 2014; Seo et al., 2014).

Here, several illustrative examples are presented to demonstrate the utility of $2 \mathrm{DCOS}$ in polymer studies. Special techniques in $2 \mathrm{DCOS}$, such as hetero-spectral correlation, positive null-space projection, and 2DOCS combined with chemometric methods are discussed.

\section{D IR Correlation Spectroscopy}

In the field of polymer study through 2DCOS, IR spectroscopy is the most commonly used analytical probes. The advantages of 2DCOS, such as the enhanced spectral resolution and determination of sequential changes of spectral band intensities, can provide the useful information in characterizing structural changes of polymer obtained as a function of a perturbation, which is not readily observed in the conventional spectroscopy (Kim et al., 2006b; Huang et al., 2009; Unger et al., 2009, 2011; Del Río et al., 2010; Huang and Kuo, 2010; Jelèić et al., 2010; Jia et al., 2010; Lee et al., 2010, 2012; Peng et al., 2010; Popescu and Vasile, 2010, 2011; Zheng et al., 2010; Cheng et al., 2011; Jin et al., 2011; Kuo and Liu, 2011; Wang and $\mathrm{Wu}, 2011$; Zhang et al., 2011; Ando et al., 2012; Qu et al., 2012; Su et al., 2012; Wu et al., 2012; Chai et al., 2013; Lai and Wu, 2013; Park et al., 2013; Shinzawa et al., 2013; Wang et al., 2013, 2014; Hou et al., 2014; Noda, 2014c; Seo et al., 2014). 
Popular perturbations in polymer studies are temperature (Kim et al., 2006b; Unger et al., 2009, 2011; Jia et al., 2010; Peng et al., 2010; Popescu and Vasile, 2010; Tang et al., 2010; Zheng et al., 2010; Cheng et al., 2011; Wang and Wu, 2011; Su et al., 2012; Wang et al., 2013, 2014; Hoshina et al., 2014; Hou et al., 2014; Seo et al., 2014), concentration (Huang et al., 2009; Jelèić et al., 2010; Kuo and Liu, 2011; Popescu and Vasile, 2011; Wu et al., 2012; Grzeszczuk et al., 2013), time (Huang and Kuo, 2010; Jin et al., 2011; Ando et al., 2012; Lee et al., 2012; Qu et al., 2012; Su et al., 2012; Lai and Wu, 2013; Park et al., 2013; Noda, 2014c), and pressure (Zhang et al., 2011; Shinzawa et al., 2013), etc. Especially, temperature is the most commonly used because ordinary changes in density, which give rise to nonspecific spectral changes, usually are accompanied by structural changes in polymer upon heating or cooling.

Choi et al. demonstrated the details of thermal behavior of spin-coated films of biodegradable poly(3-hydroxybutyrateco-3-hydroxyhexanoate) or $\mathrm{P}(\mathrm{HB}-\mathrm{co}-\mathrm{HHx})(\mathrm{HHx}=12.0$, $10.0,3.8 \mathrm{~mol} \%$ ) copolymers by using 2DCOS (Choi et al., 2010). The temperature-dependent infrared-reflection absorption (IRRAS) spectra of a spin-coated film of $\mathrm{P}(\mathrm{HB}-\mathrm{co}-\mathrm{HHx})$ $(\mathrm{HHx}=12.0 \mathrm{~mol} \%)$ copolymer, which were measured during the heating process, are shown in Figure 2. Two distinct $\mathrm{C}=\mathrm{O}$ stretching bands, a crystalline band and an amorphous band are observed respectively at $1726 \mathrm{~cm}^{-1}$ and near $1751 \mathrm{~cm}^{-1}$. The 2D correlation spectra for the $\mathrm{C}=\mathrm{O}$ stretching bands are shown in Figure 3. Two main bands are observed at 1726 and $1751 \mathrm{~cm}^{-1}$ assigned to the crystalline band and the amorphous band, respectively, in the synchronous $2 \mathrm{D}$ correlation spectrum. Interestingly the crystalline band at $1726 \mathrm{~cm}^{-1}$ observed in synchronous $2 \mathrm{D}$ correlation spectrum is clearly resolved into two bands at 1721 and $1730 \mathrm{~cm}^{-1}$ in the asynchronous $2 \mathrm{D}$ correlation spectrum, which is hardly detectable in the original IRRAS spectra shown in Figure 2. A band observed at a lower wavenumber corresponds to the well-ordered primary crystals and the other at a higher wavenumber corresponds to less ordered secondary crystals. From the analysis of the sign of cross peaks in $2 \mathrm{D}$ correlation spectra, they determined the sequential order of spectral changes with increasing temperature that the intensity of an amorphous band changes first and then that for less ordered secondary crystals changes before that for well-ordered secondary crystals.

The application of 2DCOS in the analysis of transport phenomena in polymers (Musto et al., 2011; Lai and Wu, 2013; Galizia et al., 2014) can provide useful insights about the distribution of penetrant in polymer matrix, which is of great practical relevance in several applications, such as membrane science, drug delivery, and polymer durability. Musto et al. applied 2D IR correlation spectroscopy for the investigation of the diffusion process and the sorption equilibrium of water vapor in polyimide films (Musto et al., 2011). The molecular level characterization of the mass-transport process and the sorption thermodynamics were detected. They also investigated the diffusion mechanism in biocompatible thermoplastic polymer, poly-e-caprolactone (PCL), by using 2D IR correlation spectroscopy, and the sorption-desorption cycle for a molecular level characterization of the $\mathrm{H}_{2} \mathrm{O} / \mathrm{PCL}$ system and molecular interaction formed (H-bonding) were detected (Galizia et al.,

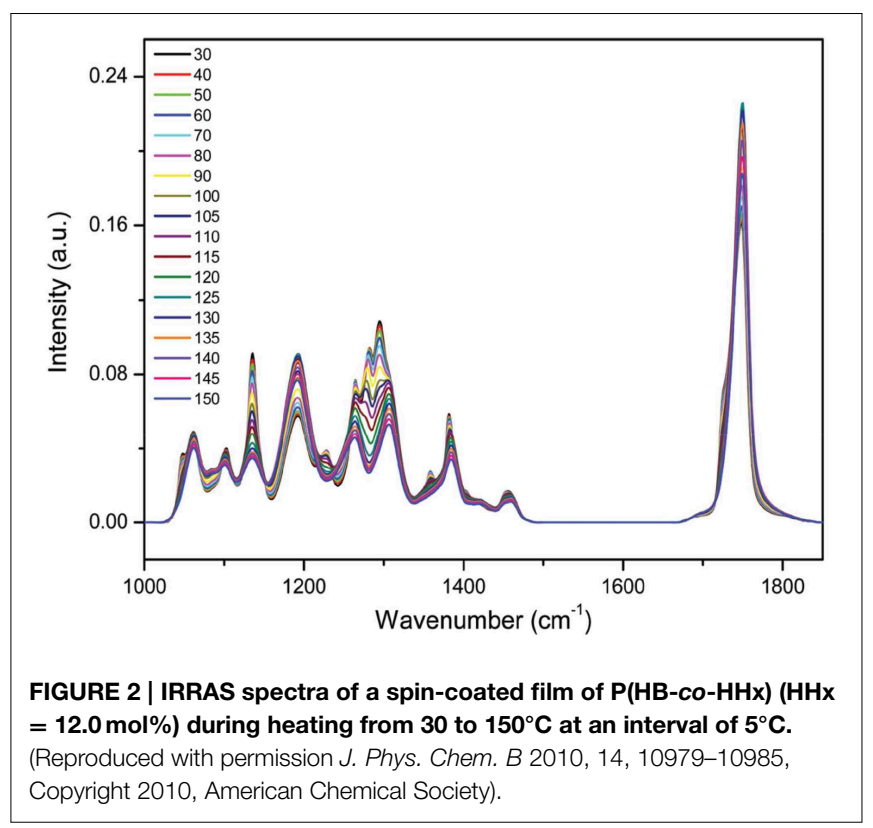

2014). Lai and $\mathrm{Wu}$ reported the water sorption and desorption processes with a pharmaceutical amphiphilic copolymer poly(3(2-methoxyethyl)-N-vinyl-2-pyrrolidone) investigated by using 2D IR correlation spectroscopy (Lai and $\mathrm{Wu}, 2013$ ). Different states of hydrogen-bonded water molecules were also detected in 2D IR correlation spectra.

Park et al. reported the mechanism of chemical gelation process of poly( $N$-isopropylacrylamide) (PNiPAAm) hydrogel by using in situ observations with time-resolved FTIR and 2DCOS at two characteristic preparation temperatures below and above the lower critical solution temperature (LCST) of PNiPAAm aqueous solution (Park et al., 2013). Figures 4A,B show the FTIR spectra in the $1800-1050 \mathrm{~cm}^{-1}$ region for the NiPAAm gelation process measured at $T_{p}=22$ and $38^{\circ} \mathrm{C}$, respectively. The spectral changes during the NiPAAm gelation process at the two different temperatures were qualitatively similar except for the differences associated with the time required for completing the gelation reaction $\left(\sim 30\right.$ and $\sim 15 \mathrm{~min}$ at $T_{p}=22$ and $38^{\circ} \mathrm{C}$, respectively). The bands at 1628 (Figure 4A) and $1630 \mathrm{~cm}^{-1}$ (Figure 4B) started to appear at $\sim 20$ and $\sim 8 \mathrm{~min}$ after the onset of the reaction, respectively. Those bands became increasingly remarkable with time and most prominent at the end of the gelation process, independent of $T_{p} s$. It identifies the specific time-spans for two stage reaction process: the first-stage giving rise to linear and branched random copolymers of NiPAAm and cross-linker monomers and the second-stage giving rise to crosslinking into macroscopic network structure. 2DCOS was thus applied both the first-stage and second-stage reaction processes to better elucidate the gelation process. The each stage of $2 \mathrm{D}$ correlation spectra for the NiPAAm gelation process at 22 and $38^{\circ} \mathrm{C}$, respectively, shown in Figures 5, 6 are completely different, although IR spectra obtained below and above LCST are apparently similar. From the analysis of 2D correlation spectra, they firstly identified the specific time span for each stage of 


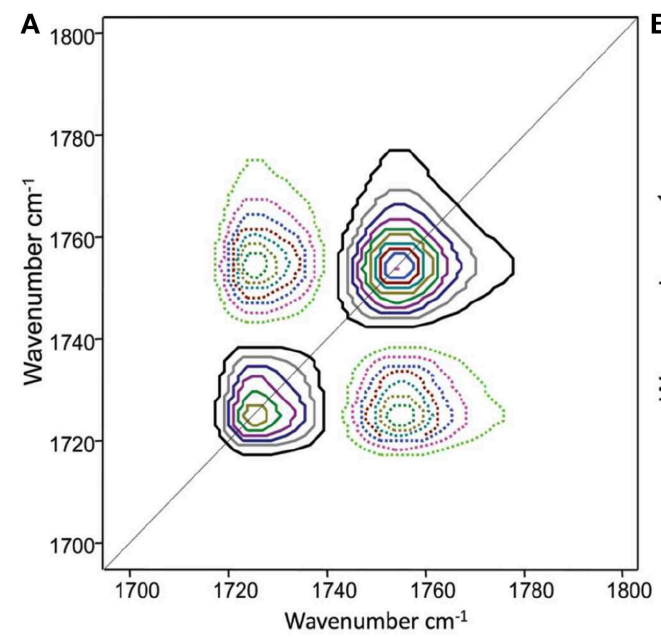

FIGURE 3 | Synchronous (A) and asynchronous (B) 2D correlation spectra obtained from the temperature-dependent IRRAS spectra of a spin-coated film of $\mathrm{P}(\mathrm{HB}-\mathrm{co}-\mathrm{HHx})(\mathbf{H H x}=\mathbf{1 2 . 0} \mathrm{mol} \%)$. The solid and

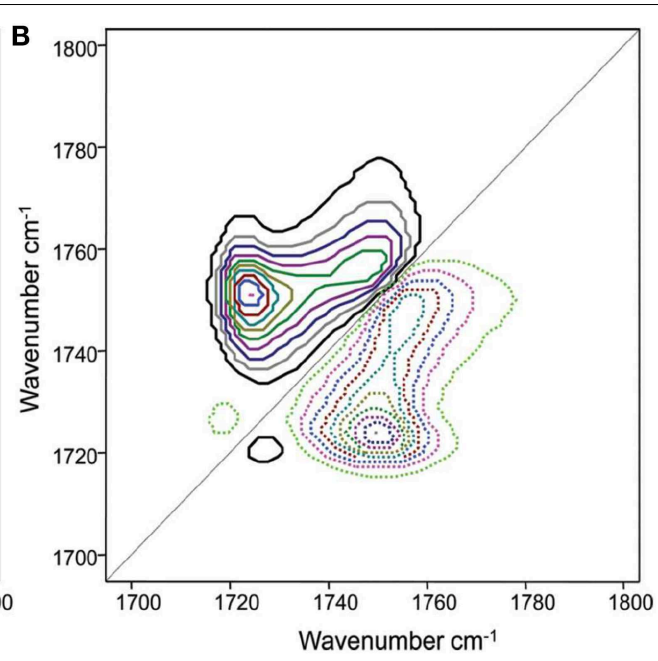

dashed lines represent positive and negative cross peaks, respectively. (Reproduced with permission J. Phys. Chem. B 2010, 14, 10979-10985, Copyright 2010, American Chemical Society).

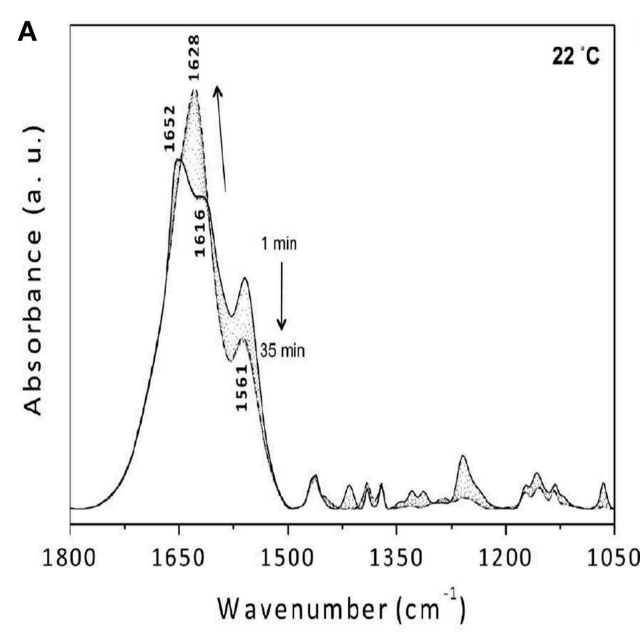

FIGURE 4 | ATR-FTIR spectra of the NiPAAm gelation process measured at $22(\mathbf{A})$ and $38^{\circ} \mathbf{C}(\mathbf{B})$. Spectra are collected at every $1 \mathrm{~min}$. The solid and dashed lines represent, respectively, the spectrum measured at 1 and $35 \mathrm{~min}$ (A) and 1 and $15 \mathrm{~min}$ (B) after initiation of the gelation reaction. The dotted lines represent the time evolution of the spectra in between the

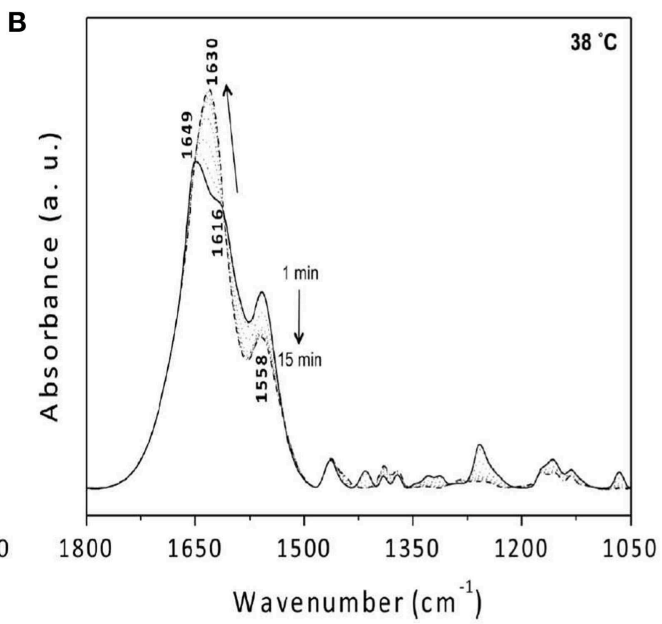

first and last spectra, and the arrows indicate the trend for the band intensity change with the upward arrow and the downward arrow showing the intensity increase and decrease with time, respectively. (Reproduced with permission Macromolecules 2013, 46, 3587-3602, Copyright 2013, American Chemical Society). the two-stage reaction process at two temperatures. Two different gelation process below and above LCST are summarized in Tables 1, 2.

\section{D Hetero-Spectral Correlation Analysis}

The hetero-correlation analysis provides a very powerful advantage to 2DCOS. Three possible possibilities in $2 \mathrm{D}$ hetero-correlation analysis are hetero-spectral correlation (Kim et al., 2006a; Choi et al., 2010; Katayama et al., 2010; Smirnova et al., 2011; Park et al., 2012; Ryu et al., 2012; Shinzawa et al., 2012), hetero-perturbation (or hybrid) correlation (Wu et al., 2002, 2006), and hetero-sample correlation
(Czarnik-Matusewicz et al., 2009; Pi et al., 2010). Among them, $2 \mathrm{D}$ hetero-spectral correlation is the most active field in applications of $2 \mathrm{D}$ hetero-correlation analysis. It can compare two completely different types of spectral data obtained for a system under a similar external perturbation. In the $2 \mathrm{D}$ hetero-spectral correlation analysis, the correlation between different spectral signals under the same perturbation can be detected. It is possible to apply 2D hetero-spectral analysis to the correlation not only between closely related spectroscopic measurement, such as IR and Raman spectra, but also between completely different types of spectroscopic or physical techniques, such as IR and X-ray spectroscopy. 


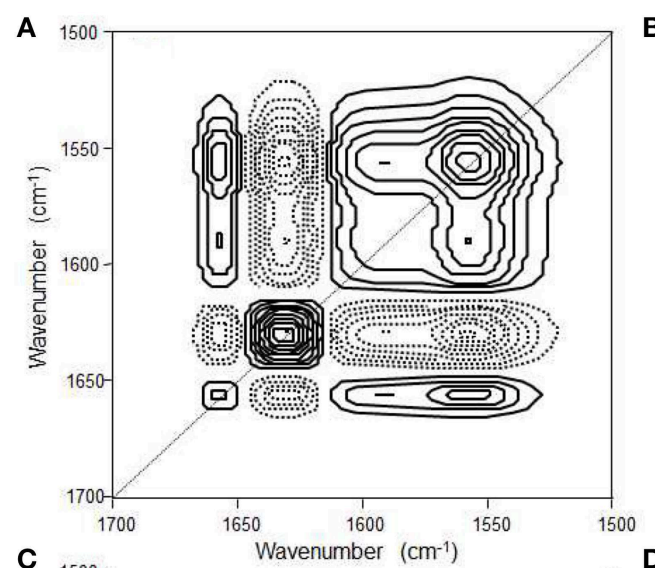

C

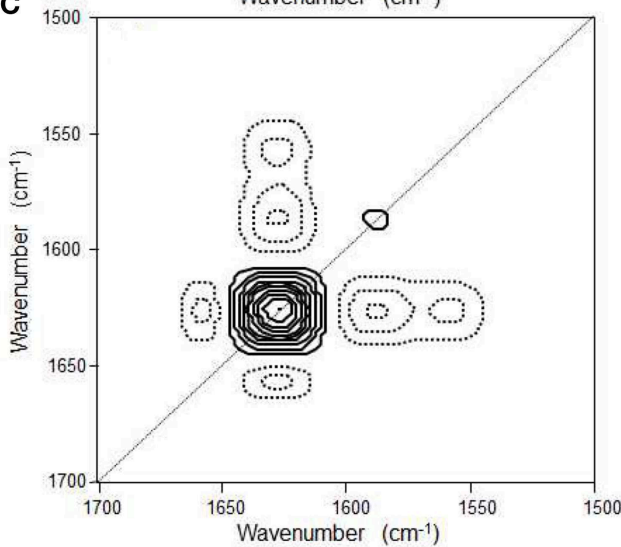

FIGURE 5 | Synchronous (A) and asynchronous (B) 2D correlation spectra in the first-stage reaction process and synchronous (C) and asynchronous (D) 2D correlation spectra in the second-stage reaction process of the NiPAAm gelation process
B
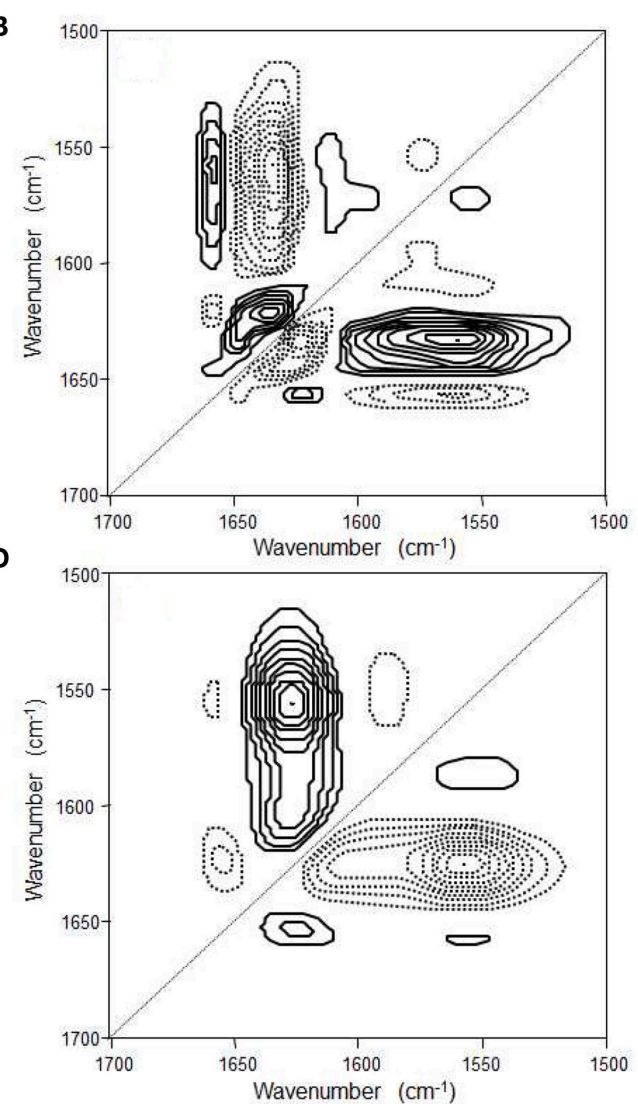

at $22^{\circ} \mathbf{C}$. The solid and dashed lines represent positive and negative cross peaks, respectively. (Reproduced with permission Macromolecules 2013, 46, 3587-3602, Copyright 2013, American Chemical Society).
Choi et al. used 2D hetero-spectral correlation between completely different types of spectroscopy, such as IR and Xray photoelectron spectroscopy (XPS), for the investigation of thermal behavior of biodegradable copolymers under increasing temperature (Choi et al., 2010).The 2D hetero-spectral IR/XPS correlation spectra of spin-coated film of $\mathrm{P}(\mathrm{HB}-\mathrm{co}-\mathrm{HHx})$ copolymer during heating process are shown in Figure 7. In synchronous 2D hetero-spectral IR/XPS correlation spectrum, two XPS band at $289.3 \mathrm{eV}$ and near $288.3 \mathrm{eV}$, which are assigned to amorphous and crystalline components, respectively, are clearly observed. Very interestingly, asynchronous 2D hetero-spectral IR/XPS correlation spectrum reveals the sequential order of the intensity changes that spectral intensity changes detected by the IR probe always occurred earlier than those by XPS. This result provides that the thermal phase transition of $\mathrm{P}\left(\mathrm{HB}-\mathrm{co}^{-}\right.$ $\mathrm{HHx}$ ) copolymer actually involves different level of microscopic scales. That is because IR probe detects long range molecular interactions while XPS detects more localized structure changes during the gradual melting process. This probe-dependent asynchronicity, which is spectral changes of IR probe appear first before those of XPS, clearly reflects the subtle difference in the selectivity and specificity of these probes toward molecular scale changes under the same external perturbation. The 2D heterospectral IR/XPS correlation analysis sheds light on the correlation between IR and XPS spectral changes, which is difficult to detect from a simple analysis of IR or XPS spectra alone.

\section{Projection 2D Correlation Analysis}

Noda has proposed a new generation technique of 2DCOS (Noda, 2010). Projection 2D correlation analysis can dramatically simplify highly congested 2D correlation spectra often encountered. This technique is based on the use of mathematical matrix projection to selectively filter out the unwanted portion of the information of spectral data. The combination of the projection and null-space projection operations might be a very useful technique to augment or attenuate select features within congested 2D correlation spectra for easier interpretation. Details of background of projection 2D analysis was previously described (Noda, 2010).

Here we will briefly provide the basic concept of projection $2 \mathrm{D}$ correlation spectra which is based on terms of a series of matrix manipulations (Noda, 2010). In generalized 2DCOS, spectral data matrix $\mathbf{A}(m \times m)$ consisting of $m$ rows of spectra with $n$ columns of spectral variables, like wavenumber, represents the 

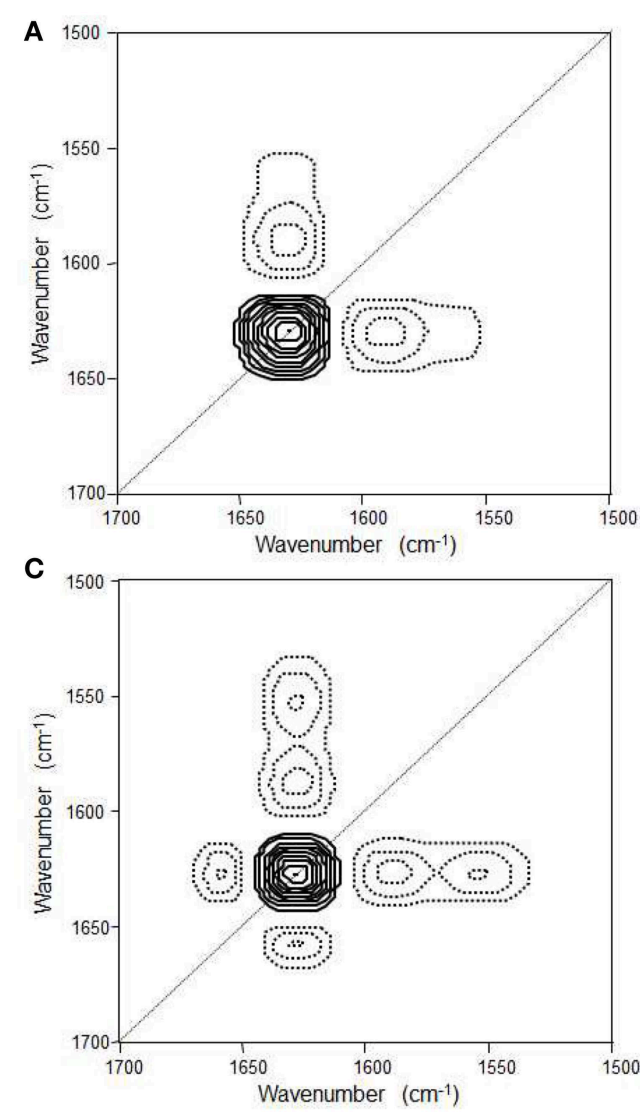

FIGURE 6 | Synchronous (A) and asynchronous (B) 2D correlation spectra in the first-stage reaction process and synchronous (C) and asynchronous (D) 2D correlation spectra in the second-stage reaction process of the NiPAAm gelation

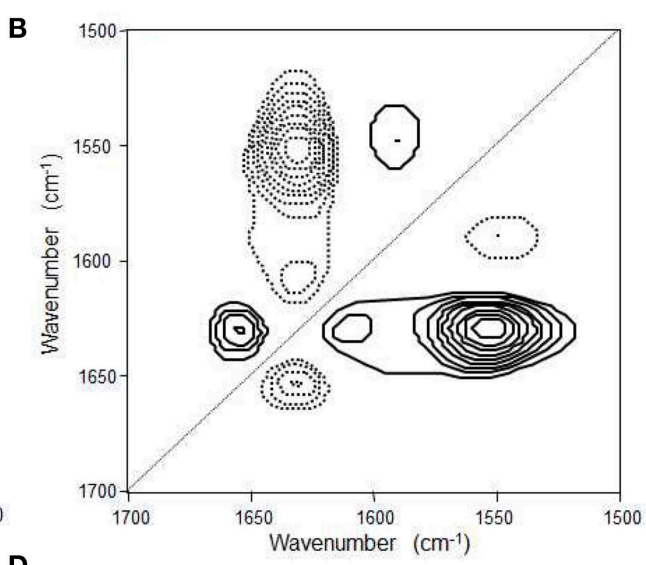

D

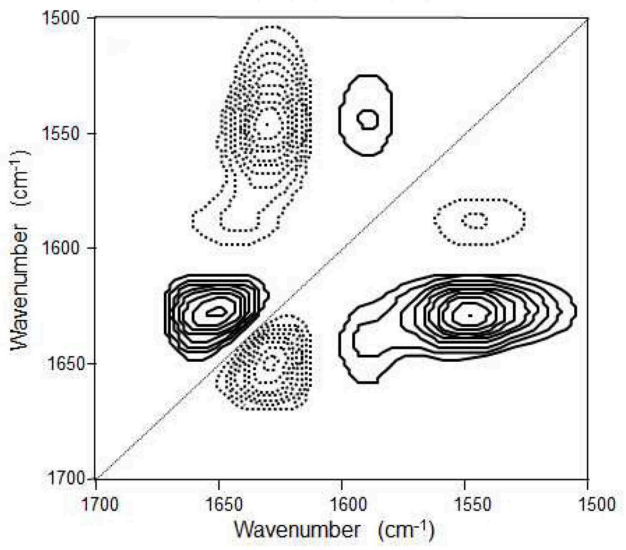

process at $38^{\circ} \mathbf{C}$. The solid and dashed lines represent positive and negative cross peaks, respectively. (Reproduced with permission Macromolecules 2013, 46, 3587-3602, Copyright 2013, American Chemical Society). system response. For convenience, each column of the matrix $\mathbf{A}$ corresponds to dynamic spectrum $\tilde{A}\left(v_{j}, t_{i}\right)$ used in Equations (3) and (4) scaled by square root of $1 /(m-1)$. Generalized $2 \mathrm{D}$ correlation spectra can be obtained by a simple matrix multiplication applied toward the spectral data matrix. The synchronous and asynchronous correlation spectra, $\boldsymbol{\Phi}$ and $\Psi$, are then obtained as

$$
\begin{array}{r}
\Phi=\mathrm{A}^{\mathrm{T}} \mathrm{A} \\
\Psi=\mathrm{A}^{\mathrm{T}} \mathrm{NA}
\end{array}
$$

As already indicated in Equation (5), $\mathbf{N}$ is the so-called HilbertNoda transformation matrix.

In projection analysis, an arbitrary $m \times m$ matrix $\mathbf{Y}$, which is different from the spectral data matrix $\mathbf{A}$, define the projection matrix $\mathbf{R}_{\mathrm{Y}}$ of $\mathbf{Y}$ as

$$
\mathrm{R}_{\mathrm{Y}}=\mathrm{Y}\left(\mathrm{Y}^{\mathrm{T}} \mathrm{Y}\right)^{-1} \mathrm{Y}^{\mathrm{T}}
$$

The superscripts ${ }^{\mathrm{T}}$ and ${ }^{-1}$, respectively, stand for the transpose and inverse operation of the matrix.
The spectral data matrix $\mathbf{A}$ can be transformed to a new form of data matrix by the projection operation. The projected data matrix $\mathbf{A}_{\mathrm{P}}$ is obtained by the simple multiplication of $\mathbf{R}_{\mathrm{Y}}$ with $\mathbf{A}$,

$$
\mathrm{A}_{\mathrm{P}}=\mathrm{R}_{\mathrm{Y}} \mathrm{A}
$$

The newly obtained projected data matrix $\mathbf{A}_{\mathrm{P}}$ represents the matrix projection of $\mathbf{A}$ onto an abstract mathematical space spanned by the columns of $\mathbf{Y}$. In other words, $\mathbf{A}_{\mathrm{P}}$ is the closest possible reconstruction of $\mathbf{A}$ by using only the linear combinations of all the columns of $\mathbf{Y}$. To make this operation possible, matrices $\mathbf{A}$ and $\mathbf{Y}$ must have the same number of rows $m$. It is actually common to select $\mathbf{Y}$ from several select columns of $\mathbf{A}$.

The corresponding null-space projection is carried out as

$$
\mathrm{A}_{\mathrm{N}}=\left(\mathrm{I}-\mathrm{R}_{\mathrm{Y}}\right) \mathrm{A}=\mathrm{A}-\mathrm{A}_{\mathrm{P}}
$$

The null-space projected data matrix $\mathbf{A}_{\mathrm{N}}$ represents the projection of $\mathbf{A}$ onto the space spanned by vectors which are orthogonal to the columns of $\mathbf{Y}$. In other words, $\mathbf{A}_{\mathrm{N}}$ is the residual after the removal of $\mathbf{A}_{\mathrm{P}}$ from $\mathbf{A}$. Thus, the projection operations separate the original data into two orthogonal parts, $\mathbf{A}=$ 
TABLE 1 | Molecular significance of sequence changes of the spectroscopic events with time for two-stage chemical gelation processes below LCST $\left(T_{p}=22^{\circ} \mathrm{C}\right)$ as observed by the 2D IR correlation spectroscopy.

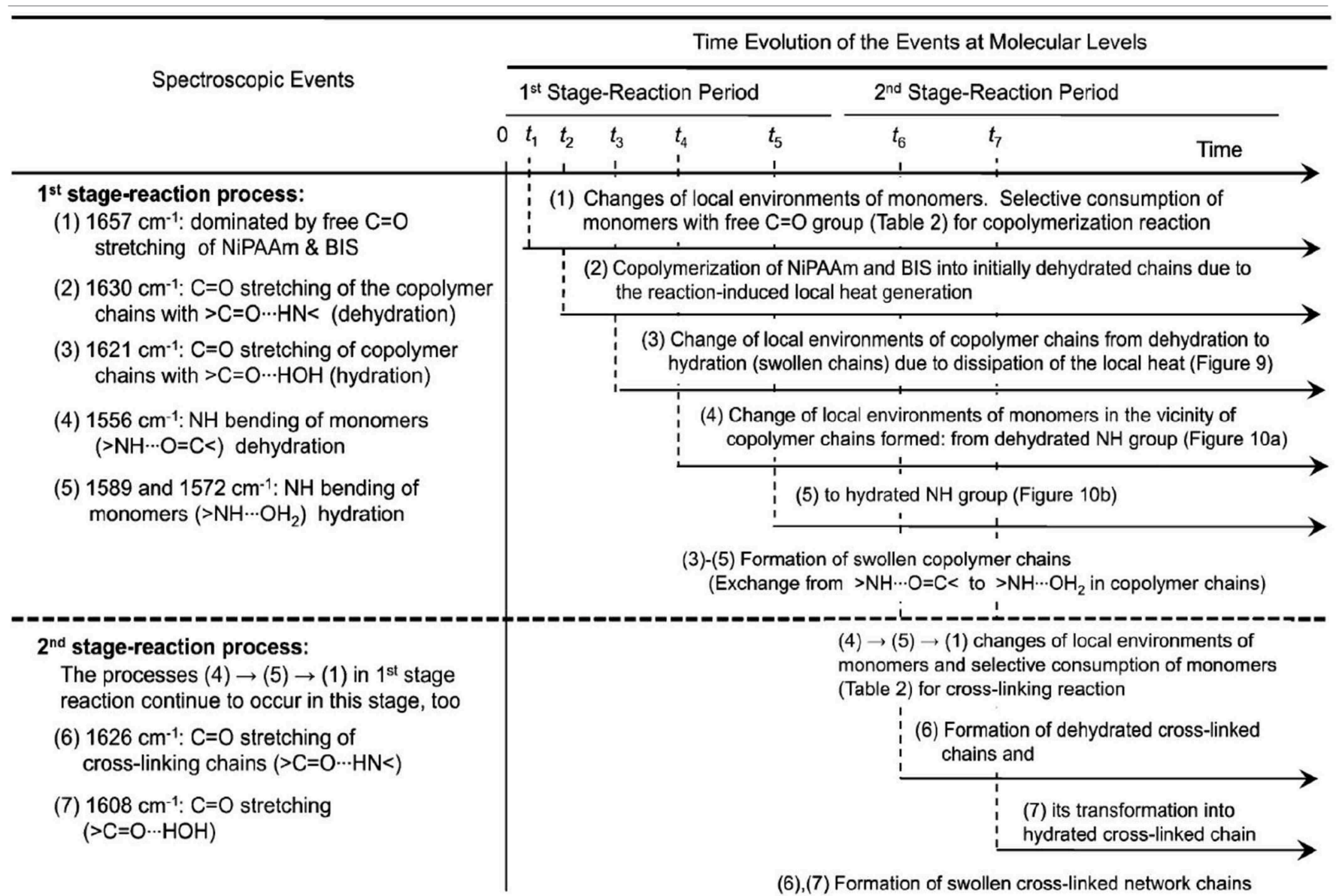

Reproduced with permission Macromolecules 2013, 46, 3587-3602, Copyright 2013, American Chemical Society.

$\mathbf{A}_{\mathrm{P}}+\mathbf{A}_{\mathrm{N}}$, by using the information contained within the chosen matrix $\mathbf{Y}$

Data matrices created by various projection-based transformation operations discussed above can be readily converted to $2 \mathrm{D}$ correlation spectra. For example, by using Equation (9), it is possible to obtain the $2 \mathrm{D}$ correlation spectra for the projected data matrix $\mathbf{A}_{\mathbf{P}}$

$$
\begin{gathered}
\Phi_{p}=A_{P}{ }^{T} A_{P}=A^{T} R_{Y} A \\
\Psi_{p}=A_{P}{ }^{T} N A_{P}=A^{T} R_{Y} N R_{Y} A
\end{gathered}
$$

The term $\mathbf{R}_{Y}$ appears in Equation (11) only once because this matrix is idempotent. 2D correlation spectra $\boldsymbol{\Phi}_{\mathrm{p}}$ and $\Psi_{\mathrm{p}}$ for the projected data provide the correlation information among select signals of $\mathbf{A}$, which are in turn correlated with the projector matrix Y. In other words, all other signals not correlated $\mathbf{Y}$ will be filtered out prior to the $2 \mathrm{D}$ correlation analysis.
Correlation analysis of the null-space projected data, $\mathbf{A}_{\mathrm{N}}=\mathbf{A}-\mathbf{A}_{\mathrm{P}}$, results in the following set of $2 \mathrm{D}$ spectra.

$$
\begin{gathered}
\Phi_{\mathrm{N}}=\mathrm{A}_{\mathrm{N}}^{\mathrm{T}} \mathrm{A}_{\mathrm{N}}=\mathrm{A}^{\mathrm{T}}\left(\mathrm{I}-\mathrm{R}_{\mathrm{Y}}\right) \mathrm{A} \\
\Psi_{\mathrm{N}}=\mathrm{A}_{\mathrm{N}}^{\mathrm{T}} \mathrm{NA}_{\mathrm{N}}=\mathrm{A}^{\mathrm{T}}\left(\mathrm{I}-\mathrm{R}_{\mathrm{Y}} \mathrm{NR}_{\mathrm{Y}}-\mathrm{NR}_{\mathrm{Y}}-\mathrm{R}_{\mathrm{Y}} \mathrm{N}\right) \mathrm{A}
\end{gathered}
$$

Kim et al. (2012) reported the dominant crystalline contribution in biodegradable polymer blend with temperature increase was successfully filtered out by using the null-space projection, which can extract other finer details. Figure 8 shows the conventional synchronous 2D correlation spectra of spin-coated film of poly(3-hydroxybutyrate-co3-hydroxyhexanoate)/polyethylene glycol (P(HB-co-HHx)/PEG) blend. In conventional $2 \mathrm{D}$ correlation spectra, all spectral changes are contributed from not PEG but $\mathrm{P}(\mathrm{HB}-\mathrm{co}-\mathrm{HHx})$ in $\mathrm{P}(\mathrm{HB}-\mathrm{co}-\mathrm{HHx}) / \mathrm{PEG}$ blend during heating process. They performed null-space projection $2 \mathrm{D}$ correlation analysis to selectively filter out the contribution of $\mathrm{P}(\mathrm{HB}-\mathrm{co}-\mathrm{HHx})$. As shown in Figure 9, the synchronous null-space projection $2 \mathrm{D}$ correlation spectra, which are constructed from the null-space projected data 
TABLE 2 | Molecular significance of sequence changes of the spectroscopic events with time for two-stage chemical gelation processes above LCST $\left(T_{p}=38^{\circ} \mathrm{C}\right)$ as observed by the 2D IR correlation spectroscopy.

Time Evolution

\begin{tabular}{l} 
Spectroscopic Events \\
\hline $1^{\text {st }}$ stage-reaction process: \\
(1) $1632 \mathrm{~cm}^{-1}: \mathrm{C}=\mathrm{O}$ stretching of \\
copolymer chain with $>\mathrm{C}=\mathrm{O} \cdots \mathrm{HN}<$ \\
(2) $1591 \mathrm{~cm}^{-1}:$ hydration $\left(>\mathrm{NH} \cdots \mathrm{OH}_{2}\right)$ \\
of monomers \\
(3) $1554 \mathrm{~cm}^{-1}:$ dehydration \\
$(>\mathrm{NH} \cdots \mathrm{O}=\mathrm{C}<$ ) of monomers \\
(4) $1657 \mathrm{~cm}^{-1}:$ dominated by free \\
$\mathrm{C}=\mathrm{O} \mathrm{stretching} \mathrm{of} \mathrm{monomers}$
\end{tabular}

\begin{tabular}{ccccc}
\hline \multicolumn{4}{c}{$1^{\text {st }}$} & \multicolumn{3}{c}{ Stage Reaction Period } \\
\cline { 2 - 5 } & $t_{1}$ & $t_{2}$ & $t_{3}$ & $t_{4}$ \\
& $\vdots$ & 1 & 1 & 1 \\
& $\vdots$ & $!$ & $!$ & $!$
\end{tabular}

(1) Copolymerization into dehydrated chains to form globular chains: No selective consumption of monomers for copolymerization (Table 2) (2) Change in local environment of monomers in the vicinity of copolymers from hydrated $\mathrm{NH}$ groups (Figure 11a)

(3) to dehydrated NH groups (Figure 11b)

(4) A further change in local environments of monomers in the vicinity of copolymer chains induced by the local phase separation of globular chains $\mathrm{G}$ into droplets (Scheme 1c) and selective sonsumption of monomers for copolymerization within the domains $D$

\section{$2^{\text {nd }}$ stage-reaction process:}

(5) $1626 \mathrm{~cm}^{-1}: \mathrm{C}=\mathrm{O}$ stretching of cross-linked chains $(>\mathrm{C}=\mathrm{O} \cdots \mathrm{HN}<)$

(6) $1589 \mathrm{~cm}^{-1}$ : hydrated $\mathrm{NH}$ groups of monomers $\left(>\mathrm{NH} \cdots \mathrm{OH}_{2}\right)$

(7) $1546 \mathrm{~cm}^{-1}$ : dehydrated $\mathrm{NH}$ groups of monomers $(>\mathrm{NH} \cdots \mathrm{O}=\mathrm{C}<$ )

(8) $1650 \mathrm{~cm}^{-1}$ : dominated by free $\mathrm{C}=\mathrm{O}$ stretching of monomers
(5) Cross-linking within phase-separated droplets to form microgels (Scheme 1e)

(6) Change of local environments of monomers in the vicinity of polymers from hydrated monomers

(7) to dehydrated monomers
accompanied by the macro-
phase separation as illusrated
from Scheme 1(e) to 1(f)

(8) Selective consumption of monomers with free $\mathrm{C}=\mathrm{O}$ group for cross-linking within the dehydrated sponge-like domain into macrogel (Scheme 1f)

Reproduced with permission Macromolecules 2013, 46, 3587-3602, Copyright 2013, American Chemical Society.

with the crystalline signals of $\mathrm{P}(\mathrm{HB}-\mathrm{co}-\mathrm{HHx})$ removed, are completely different with conventional $2 \mathrm{D}$ correlation spectra. The observed new bands at 1313, 1105, and $1065 \mathrm{~cm}^{-1}$ in Figure 9A, which are hardly detected in conventional $2 \mathrm{D}$ correlation spectrum, can be assigned to PEG. In Figure 9B, two bands at 2972 and $2875 \mathrm{~cm}^{-1}$, which are not observed in conventional $2 \mathrm{D}$ correlation spectrum, can also be assigned to PEG. The subtle contribution of $\mathrm{PEG}$ in spin-coated film of $\mathrm{P}(\mathrm{HB}-\mathrm{co}-\mathrm{HHx}) / \mathrm{PEG}$ blend during heating process is clearly detected in null-space $2 \mathrm{D}$ projection correlation spectra.

\section{Combination of 2DCOS and Chemometric Techniques}

The fruitful combination of 2DCOS and chemometric techniques often provides more useful information to interpret subtle spectral changes of system, which is barely detected in conventional 2DCOS (Jung, 2004; Jung et al., 2002, 2003a,b,c,d, 2006). Jung et al. introduced the possible combination of 2DCOS and principal component analysis (PCA). In this technique named PCA 2DCOS, PCA is an essential and integral part of the subsequent 2DCOS (Jung, 2004; Jung et al., 2002). Jung et al. also reported a new concept of engenvalue manipulating transformation (EMT) for PCA 2DCOS (Jung et al., 2003b,c,d, 2006).

A brief background of PCA 2DCOS and EMT are provided here. The original set of perturbation-dependent spectral data matrix $\mathbf{A}$ is an $n \times m$ matrix with $n$ spectra and $m$ wavenumber points. In PCA, the significant part of the data matrix $\mathbf{A}^{\star}$ can be expressed as the product of score and loading matricies

$$
\mathbf{A}=\mathbf{W} \mathbf{V}^{\mathbf{T}}+\mathbf{E}=\mathbf{A}^{*}+\mathbf{E}
$$

where $\mathbf{W}$ and $\mathbf{V}$ are the loading matrix $(m \times r)$ and score matrix $(n \times r)$, respectively, and $\mathbf{E}$ is the residual matrix often related with pure noise. The matrix product $\mathbf{A}^{*}$ is the noise-free 


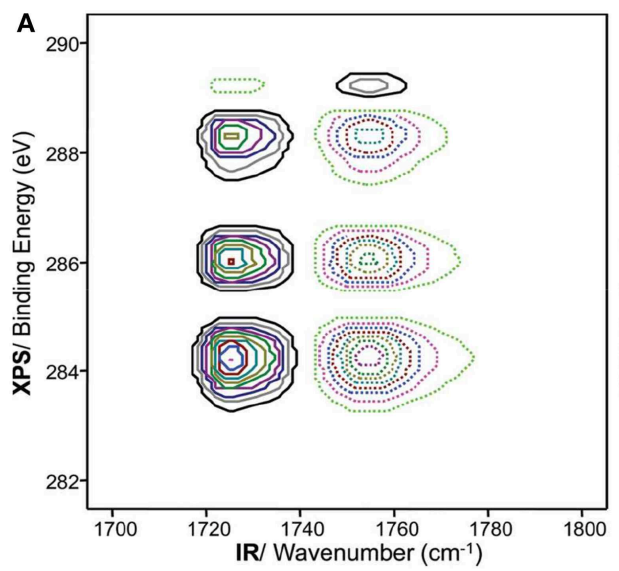

FIGURE 7 | Synchronous (A) and asynchronous (B) hetero-spectral 2D XPS/IR correlation spectrum for a spin-coated film of $\mathrm{P}(\mathrm{HB}-\mathrm{co}-\mathrm{HHx})$

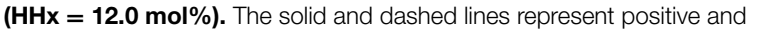

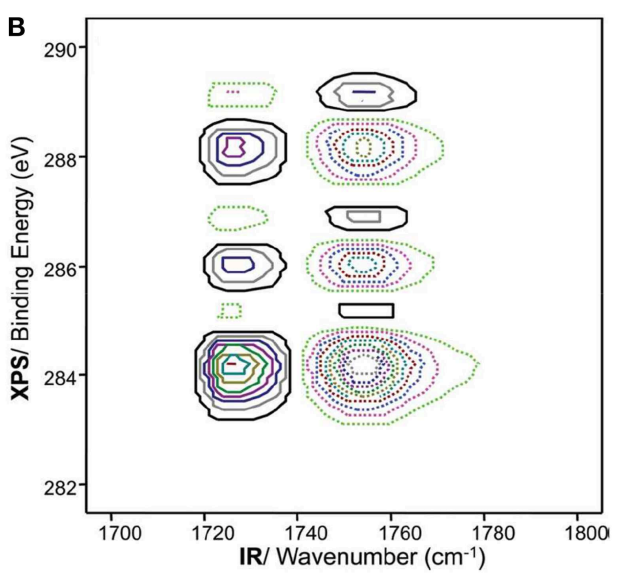

negative cross peaks, respectively. (Reproduced with permission J. Phys. Chem. B 2010, 14, 10979-10985, Copyright 2010, American Chemical Society).

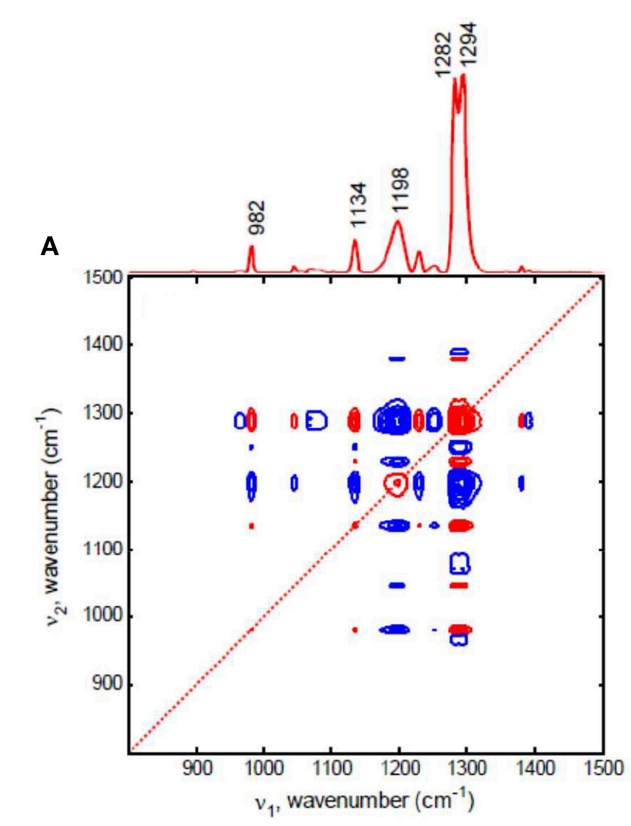

FIGURE 8 | Synchronous conventional 2D correlation spectra in the region of $\mathrm{C}-\mathrm{H}$ deformation, $\mathrm{C}-\mathrm{O}-\mathrm{C}$ stretching, and $\mathrm{C}=\mathrm{O}$ stretching modes in $1000-1500 \mathrm{~cm}^{-1}$ (A) and $\mathrm{C}-\mathrm{H}$ stretching mode in $2800-3050 \mathrm{~cm}^{-1}$ (B) for spin-coated film of

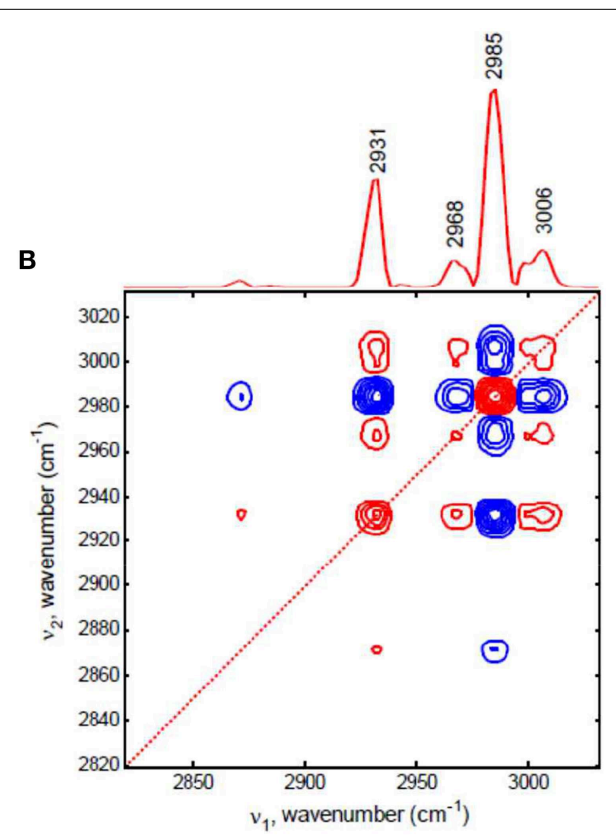

P(HB-co-HHx)/PEG blend, respectively. The red and blue lines represent positive and negative cross peaks, respectively. (Reproduced with permission Vib. Spectrosc. 2012, 60, 163-167, Copyright 2012, Elsevier). reconstructed data matrix of the original data $\mathbf{A}$.

$$
\mathbf{A}^{*}=\mathbf{W} \mathbf{V}^{\mathrm{T}}
$$

In PCA 2DCOS, this reconstructed data matrix $\mathbf{A}^{*}$ is used instead of the original data matrix. PCA 2DCOS reconstructed from a few selected significant scores and loading vectors of PCA can accentuate only the most important features of synchronicity and asynchronicity without noise contribution. It is a very powerful technique for eliminating noise contribution from the spectra to extract useful information.

The PCA-reconstructed data matrix $\mathbf{A}^{*}$ can be also expressed in the form of singular value decomposition (SVD),

$$
\mathbf{A}^{*}=\mathbf{U} \mathbf{S} \mathbf{V}^{\mathrm{T}}
$$

and

$$
S=\mathbf{L}^{1 / 2}
$$




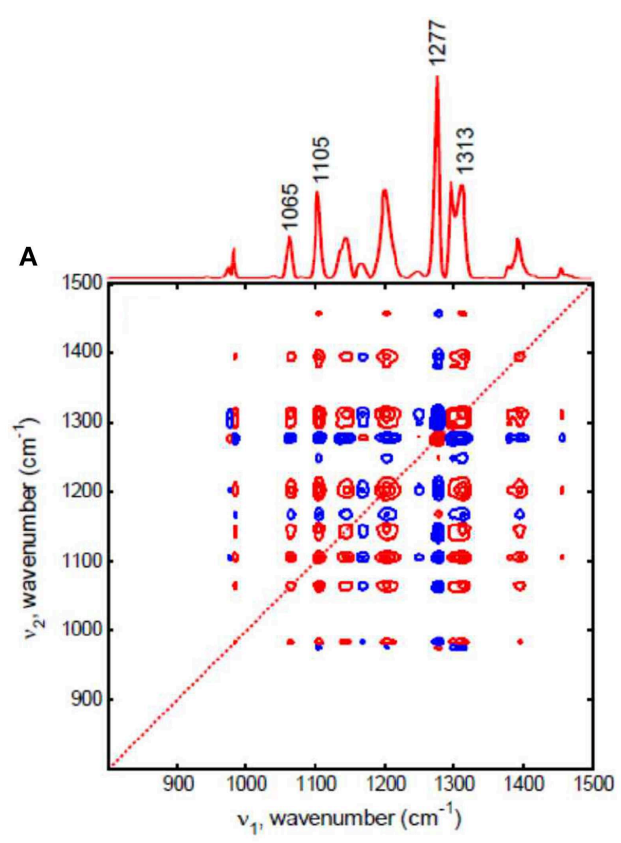

FIGURE 9 | Synchronous null-space projection 2D correlation spectra in the region of $\mathrm{C}-\mathrm{H}$ deformation, $\mathrm{C}-\mathrm{O}-\mathrm{C}$ stretching, and $\mathrm{C}=\mathrm{O}$ stretching modes in $1000-1500 \mathrm{~cm}^{-1}$ (A) and $\mathrm{C}-\mathrm{H}$ stretching mode in $2800-3050 \mathrm{~cm}^{-1}$ (B) for spin-coated film of

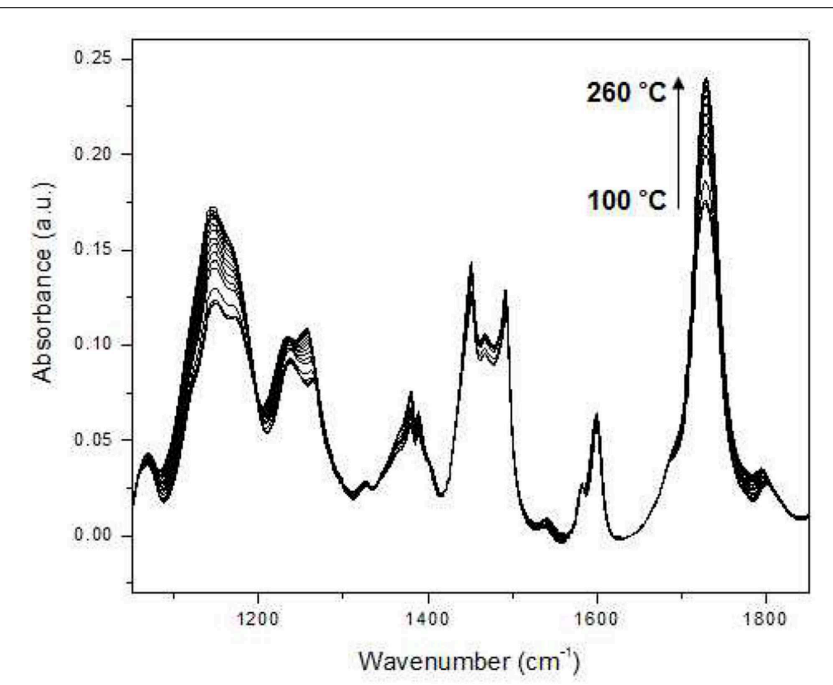

FIGURE 10 | Temperature-dependent IR spectra of polystyrene-block-poly(n-pentyl methacrylate) (PS-PnPMA) measured during heating from 100 to $260^{\circ} \mathbf{C}$ at an interval of $5^{\circ} \mathbf{C}$. (Reproduced with permission J. Mol. Struct. 2006, 799, 96-101, Copyright 2006, with permission from Elsevier).

where $\mathbf{U}$ and $\mathbf{S}$ are the orthonormal score matrix and diagonal matrix containing the singular value, respectively.

Here $\mathbf{L}=\mathbf{W}^{\prime} \mathbf{W}$ is a diagonal matrix where each diagonal element corresponds to the eigenvalue of principal component. The

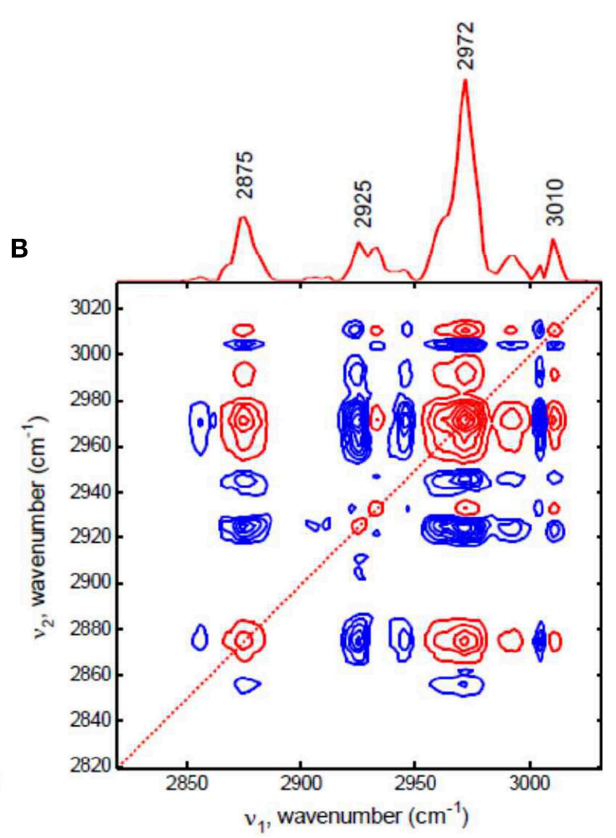

P(HB-co-HHx)/PEG blend, respectively. The red and blue lines represent positive and negative cross peaks, respectively. (Reproduced with permission Vib. Spectrosc. 2012, 60, 163-167, Copyright 2012, Elsevier). score matrix $\mathbf{W}$ is expressed in the form $\mathbf{W}=\mathbf{U} \mathbf{S}$ and can be obtained directly from $\mathbf{W}=\mathbf{A} \mathbf{V}$.

By manipulating and replacing eigenvalues of $\mathbf{A}^{*}$, the new transformed data matrix $\mathbf{A}^{* *}$ can be obtained

$$
\mathbf{A}^{* *}=\mathbf{U S ~ S}^{* *} \mathbf{V}^{\mathrm{T}}
$$

where $\mathbf{S}^{* *}$ is given by varying the corresponding eigenvalues in $\mathbf{S}$ by raising them to the power of $\mathrm{m}$.

$$
\mathrm{S}^{* *}=\mathrm{S}^{\mathrm{m}}
$$

The new EMT-reconstructed data matrix $\mathbf{A}^{* *}$ is used instead of $\mathbf{A}^{*}$ to enhance $2 \mathrm{D}$ correlation spectra. The smaller eigenvalues becomes more prominent, by uniformly lowering the power of a set of eigenvalues associated with the original data. In this technique, the contributions of minor components but potentially important factors is amplified.

Jung et al. demonstrated that PCA 2DCOS through EMT technique was performed to more clearly understand the phase behavior of polystyrene-block-poly(n-pentyl methacrylate) (PS-PnPMA) (Jung et al., 2006). PS-PnPMA is a very interesting closed-loop block copolymer, which has a lower disorder-to-order transition (LDOT) temperature and an upper order-to-disorder transition (UODT) temperature. The temperature-dependent IR spectra of PS-PnPMA measured during heating from $100-260^{\circ} \mathrm{C}$ are shown in Figure 10. In the conventional 2D IR correlation spectra shown in Figure 11, the ordered state is completely different with two disordered 

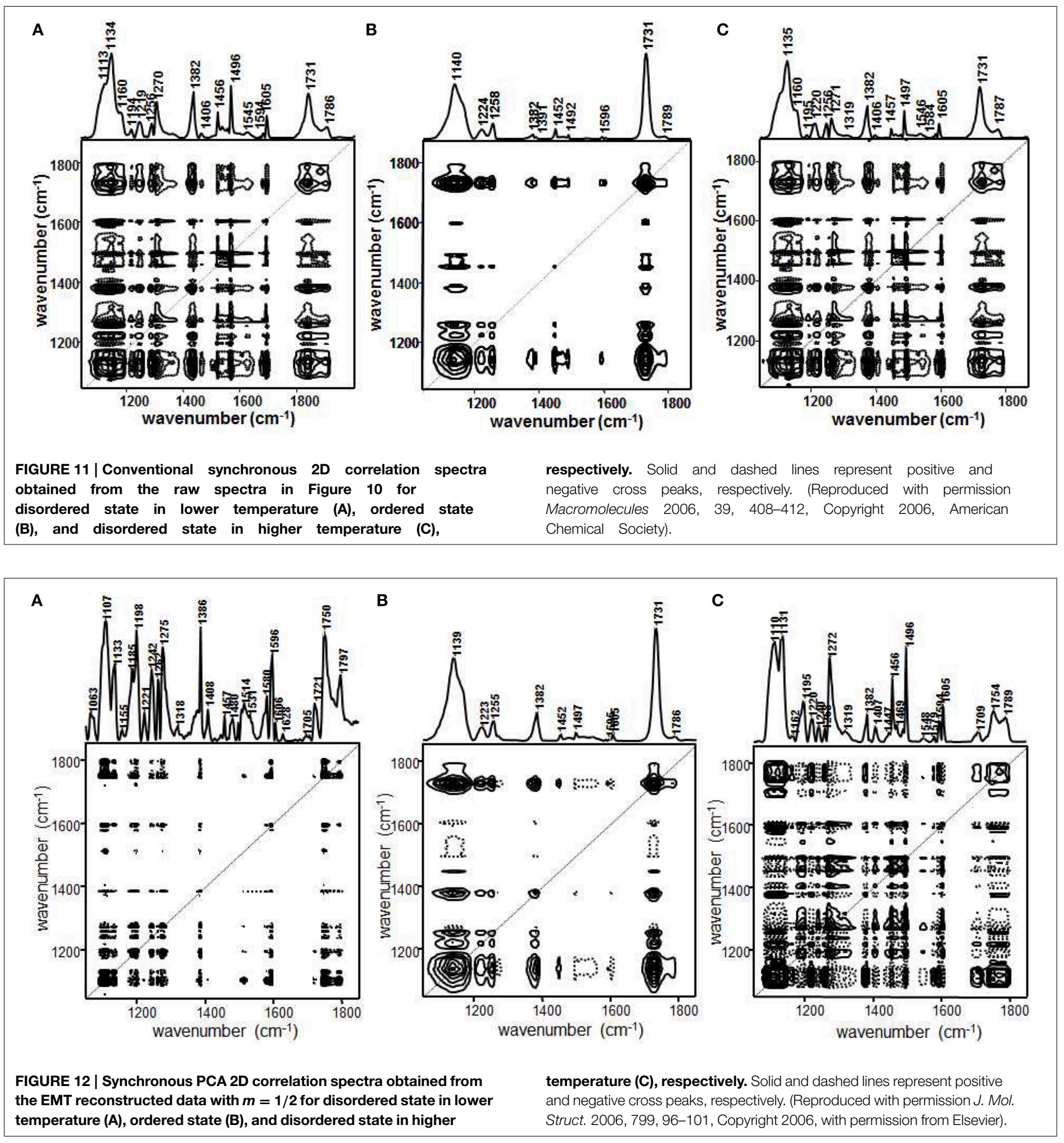

states and these two disordered states at lower and higher temperatures are also different (Kim et al., 2006b). To highlight subtle differences of the two disordered states of PSPnPMA, they applied PCA 2DCOS through EMT method to the temperature-dependent IR spectra. In PCA analysis, the original spectral data set shown in Figure 10 was decomposed into the scores and loading vectors. Synchronous PCA 2D correlation spectra generated from the reconstructed data matrix $\mathbf{A}^{*}$ with the three principal components are like the conventional 2D correlation spectra but without noise contribution. Figures 12A-C shows synchronous PCA 2D correlation spectra generated from the EMT-reconstructed spectral data matrix $\mathbf{A}^{* *}$ obtained by replacing the original eigenvalues with $m=1 / 2$ for disordered state at lower temperature, ordered state, and disordered state at 
higher temperature, respectively. By lowering the power of a set of eigenvalues associated with the original data, hidden property of phase transition from the contribution of minor but potentially interesting is much more greatly accentuated than conventional 2D correlation spectra. As shown in Figure 12, synchronous spectrum generated from the EMT-reconstructed spectral data matrix of the ordered state is completely different from those in the two disordered states and the clear difference between two disordered states is also observed. In the power spectrum, extracted along the diagonal line of the synchronous $2 \mathrm{D}$ correlation spectrum, in the top of Figures 12A,C, intensities of bands from $\mathrm{C}-\mathrm{C}-\mathrm{O}$ stretching, $\mathrm{C}-\mathrm{H}$ deformation, and $\mathrm{C}=\mathrm{O}$ stretching of PnPMA change greatly at lower temperature while those from phenyl group in PS change greatly at higher temperature. The distinct difference in two disordered states in the cross correlations of the bands from phenyl group in PS with that from C-C-O group in PnPMA reveals that the conformation of PS-PnPMA and the weak interaction between phenyl group of PS and the side chain of PnPMA in the two disordered states are different. The EMT technique clearly distinguish the very subtle differences of spectra which are not observed in conventional 2D correlation spectra.

Jung et al. also demonstrated the use of 2DCOS in conjunction with alternating least squares (ALS) based selfmodeling curve resolution (SMCR) analysis of spectral data sets (Jung et al., 2003a). In this iterative regression technique, asynchronous $2 \mathrm{D}$ correlation peaks for the identification of pure variables were used as the initial estimates in the ALS process. Choosing the most distinct bands via the positions of asynchronous $2 \mathrm{D}$ peaks is a viable starting point for ALS iteration (Jung et al., 2003a; Hong et al., 2005). Once the pure variables are selected, ALS regression can be

\section{References}

Ando, Y., Sato, H., Shinzawa, H., Okamoto, M., Noda, I., and Ozaki, Y. (2012). Isothermal melt crystallization behavior of neat poly(l-lactide) (PLLA) and PLLA/organically modified layered silicate (OMLS) nanocomposite studied by two-dimensional (2D) correlation spectroscopy. Vibrat. Spec. 60, 158-162. doi: 10.1016/j.vibspec.2012.01.021

Brewster, V. L., Ashton, L., and Goodacre, R. (2013). Monitoring guanidiniuminduced structural changes in ribonuclease proteins using raman spectroscopy and 2D correlation analysis. Anal. Chem. 85, 3570-3575. doi: $10.1021 / \mathrm{ac} 303265 \mathrm{q}$

Cerdà-Costa, N., De La Arada, I., Avilés, F. X., Arrondo, J. L. R., and Villegas, S. (2009). Influence of aggregation propensity and stability on amyloid fibril formation as studied by fourier transform infrared spectroscopy and two-dimensional COS analysis. Biochemistry 48, 10582-10590. doi: 10.1021/bi900960s

Chai, F., Chen, Y., You, Z., Xia, Z., Ge, S., Sun, Y., et al. (2013). Two Keggin-type heteropolytungstates with transition metal as a central atom: crystal structure and magnetic study with 2D-IR correlation spectroscopy. J. Solid State Chem. 202, 161-167. doi: 10.1016/j.jssc.2013. 03.051

Cheng, Y.-H., Chen, W.-P., Shen, Z., Fan, X.-H., Zhu, M.-F., and Zhou, Q.F. (2011). Influences of hydrogen bonding and peripheral chain length on mesophase structures of mesogen-jacketed liquid crystalline polymers with amide side-chain linkages. Macromolecules 44, 1429-1437. doi: $10.1021 / \mathrm{ma102444t}$ used to obtain the concentration profiles and pure component spectra.

Hong et al. studied the electrochemical polymerization of aniline by using real-time spectroelectrochemical experiments conducted concurrently with potentiodynamic scans (Hong et al., 2005). They performed 2DCOS and subsequent extraction of pure component spectra as well as their relative concentration profiles from complex spectroelectrochemical data employing the ALS-based SMCR method. All of the spectra of intermediate species proposed in the literature are identified. The concentrations of intermediate species varying as a function of the scanned potential are also determined. It was the first complete analysis of the complex spectra of aniline oxidation, which provides full understanding of the aniline polymerization reaction.

\section{Summary}

2DCOS has become a very popular tool in the field of polymer study. It can be utilized with a number of spectroscopic and other analytical probes for a very broad range of polymer systems by employing different types of external perturbations to induce spectral variations. This review covers the basic concept of generalized 2DCOS and noteworthy progress in 2DCOS and their applications in polymer study. New developments in 2DCOS provide a powerful analytical technique applicable to the in-depth analysis of various spectral data. Active and steady progress in 2DCOS would open a way for studying polymers in many applications.

\section{Acknowledgments}

This work was supported by Kangwon National University.
Choi, H. C., Ryu, S. R., Ji, H., Kim, S. B., Noda, I., and Jung, Y. M. (2010). Two-dimensional heterospectral correlation analysis of X-ray photoelectron spectra and infrared spectra for spin-coated films of biodegradable Poly(3hydroxybutyrate-co-3-hydroxyhexanoate) copolymers. J. Phys. Chem. B 114, 10979-10985. doi: 10.1021/jp103288x

Czarnik-Matusewicz, B., and Jung, Y. M. (2014). “Two-Dimensional Mid-Infrared Correlation Spectroscopy in Protein Research," in Optical Spectroscopy and Computational Methods in Biology and Medicine, ed M. Baranska (Berlin: Springer), 213-250.

Czarnik-Matusewicz, B., Kim, S. B., and Jung, Y. M. (2009). A study of ureadependent denaturation of $\beta$-lactoglobulin by principal component analysis and two-dimensional correlation spectroscopy. J. Phys. Chem. B 113, 559-566. doi: $10.1021 /$ jp808396g

Del Río, V., Spegazzini, N., Callao, M., and Larrechi, M. (2010). Spectroscopic and quantitative chemometric analysis of the epoxidised oil/amine system. J. Near Infrared Spec. 18, 281-290. doi: 10.1255/jnirs.880

Galizia, M., La Manna, P., Mensitieri, G., Pannico, M., and Musto, P. (2014). Diffusion in polymers as investigated by two-dimensional correlation spectroscopy: the H2O/PCL system. J. Mol. Struct. 1069, 290-298. doi: 10.1016/j.molstruc.2014.02.045

Grzeszczuk, M., Grañska, A., and Szostak, R. (2013). Raman spectroelectrochemistry of polyaniline synthesized using different electrolytic regimes -multivariate analysis. Int. J. Electrochem. Sci. 8, 8951-8965.

Guo, L., Spegazzini, N., Sato, H., Hashimoto, T., Masunaga, H., Sasaki, S., et al. (2011). Multistep crystallization process involving sequential formations of 
density fluctuations, "intermediate structures," and lamellar crystallites: poly(3hydroxybutyrate) as investigated by time-resolved synchrotron SAXS and WAXD. Macromolecules 45, 313-328. doi: 10.1021/ma201475t

Hong, S.-Y., Jung, Y. M., Kim, S. B., and Park, S.-M. (2005). Electrochemistry of conductive polymers. 34. two-dimensional correlation analysis of real-time spectroelectrochemical data for aniline polymerization. J. Phys. Chem. B 109, 3844-3850. doi: 10.1021/jp046218f

Hoshina, H., Ishii, S., Morisawa, Y., Sato, H., Noda, I., Ozaki, Y., et al. (2012). Isothermal crystallization of poly(3-hydroxybutyrate) studied by terahertz twodimensional correlation spectroscopy. Appl. Phys. Lett. 100, 011907(011901011903). doi: 10.1063/1.3673847

Hoshina, H., Ishii, S., and Otani, C. (2014). Separation of overlapping vibrational peaks in terahertz spectra using two-dimensional correlation spectroscopy. J. Mol. Struct. 1069, 152-156. doi: 10.1016/j.molstruc.2014.02.058

Hou, L., Ma, K., An, Z., and Wu, P. (2014). Exploring the volume phase transition behavior of POEGA- and PNIPAM-based core-shell nanogels from infraredspectral insights. Macromolecules 47, 1144-1154. doi: 10.1021/ma4021906

Huang, K.-W., and Kuo, S.-W. (2010). High-performance polybenzoxazine nanocomposites containing multifunctional POSS cores presenting vinylterminated benzoxazine groups. Macromol. Chem. Phys. 211, 2301-2311. doi: $10.1002 /$ macp. 201000362

Huang, K.-W., Tsai, L.-W., and Kuo, S.-W. (2009). Influence of octakisfunctionalized polyhedral oligomeric silsesquioxanes on the physical properties of their polymer nanocomposites. Polymer 50, 4876-4887. doi: 10.1016/j.polymer.2009.08.026

Hur, J., Jung, K.-Y., and Jung, Y. M. (2011). Characterization of spectral responses of humic substances upon UV irradiation using two-dimensional correlation spectroscopy. Water Res. 45, 2965-2974. doi: 10.1016/j.watres.2011.03.013

Izawa, K., Ogasawara, T., Masuda, H., Okabayashi, H., and Noda, I. (2001). Two-dimensional correlation gel permeation chromatography study of octyltriethoxysilane sol-gel polymerization process. Macromolecules 35, 92-96. doi: $10.1021 / \mathrm{ma} 010760+$

Jelèić, Ž., Vranješ, N., and Rek, V. (2010). Long-range processing correlation and morphological fractality of compatibilized blends of PS/ HDPE/ SEBS block copolymer. Macromol. Symp. 290, 1-14. doi: 10.1002/masy.201050401

Ji, W., Spegazzini, N., Kitahama, Y., Chen, Y., Zhao, B., and Ozaki, Y. (2012). pHresponse mechanism of $\mathrm{p}$-aminobenzenethiol on ag nanoparticles revealed by two-dimensional correlation surface-enhanced raman scattering spectroscopy. J. Phys. Chem. Lett. 3, 3204-3209. doi: 10.1021/jz301428e

Jia, L., Guo, C., Yang, L., Xiang, J., Tang, Y., Liu, C., et al. (2010). Mechanism of PEO-PPO-PEO micellization in aqueous solutions studied by twodimensional correlation FTIR spectroscopy. J. Colloid Interface Sci. 345, 332-337. doi: 10.1016/j.jcis.2010.01.060

Jiang, Y., and Wu, P. (2008). Fabrication and elimination of PTAA/P4VP layer-bylayer films. Appl. Spec. 62, 207-212. doi: 10.1366/000370208783575555

Jin, Y., Wang, W., and Su, Z. (2011). Spectroscopic study on water diffusion in poly(l-lactide)-poly(ethylene glycol) diblock copolymer film. Macromolecules 44, 2132-2139. doi: 10.1021/ma200062t

Jung, Y. M. (2004). Principal component analysis based two-dimensional correlation spectroscopy for noise filtering effect. Vib. Spec. 36, 267-270. doi: 10.1016/j.vibspec.2003.11.019

Jung, Y. M., Kim, H. J., Ryu, D. Y., Kim, S. B., and Kim, J. K. (2006). Application of principal component analysis-based two-dimensional correlation spectroscopy to characterization of order-disorder transition of polystyreneblock-poly(n-pentyl methacrylate) copolymer. J. Mol. Struct. 799, 96-101. doi: 10.1016/j.molstruc.2006.03.021

Jung, Y. M., Kim, S. B., and Noda, I. (2003a). Application of twodimensional correlation spectroscopy to chemometrics: self-modeling curve resolution analysis of spectral data sets. Appl. Spec. 57, 1376-1380. doi: $10.1366 / 000370203322554536$

Jung, Y. M., Kim, S. B., and Noda, I. (2003b). New approach to generalized two-dimensional correlation spectroscopy. II: eigenvalue manipulation transformation (EMT) for noise suppression. Appl. Spec. 57, 557-563. doi: $10.1366 / 000370203321666597$

Jung, Y. M., Kim, S. B., and Noda, I. (2003c). New approach to generalized twodimensional correlation spectroscopy. III: eigenvalue manipulation transformation (EMT) for spectral selectivity enhancement. Appl. Spec. 57, 564-570. doi: $10.1366 / 000370203321666605$
Jung, Y. M., Kim, S. B., and Noda, I. (2003d). New approach to generalized two-dimensional correlation spectroscopy. IV: eigenvalue manipulation transformation (EMT) for partial attenuation of dominant factors. Appl. Spec. 57, 850-857. doi: 10.1366/000370203322102951

Jung, Y. M., and Noda, I. (2014). “Two-dimensional correlation spectroscopy: new developments and applications," in Encyclopedia of Analytical Chemistry, ed R. A. Meyers (John Wiley \& Sons, Ltd), 1-22.

Jung, Y. M., Shin, H. S., Kim, S. B., and Noda, I. (2002). New approach to generalized two-dimensional correlation spectroscopy. 1: combination of principal component analysis and two-dimensional correlation spectroscopy. Appl. Spec. 56, 1562-1567. doi: 10.1366/000370202321116020

Katayama, N., Kondo, M., and Miyazawa, M. (2010). Study on molecular structure and hydration mechanism of Domyoji-ko starch by IR and NIR hetero 2D analysis. J. Mol. Struct. 974, 179-182. doi: 10.1016/j.molstruc.2010.02.074

Kim, H. J., Kim, S. B., Kim, J. K., and Jung, Y. M. (2006a). Two-dimensional heterospectral correlation analysis of wide-angle X-ray scattering and infrared spectroscopy for specific chemical interactions in weakly interacting block copolymers. J. Phys. Chem. B 110, 23123-23129. doi: 10.1021/jp0638282

Kim, H. J., Kim, S. B., Kim, J. K., Jung, Y. M., Ryu, D. Y., Lavery, K. A., et al. (2006b). Phase behavior of a weakly interacting block copolymer by temperature-dependent FTIR spectroscopy. Macromolecules 39, 408-412. doi: $10.1021 / \mathrm{ma} 052259 \mathrm{~d}$

Kim, M. K., Ryu, S. R., Noda, I., and Jung, Y. M. (2012). Projection 2D correlation analysis of spin-coated film of biodegradable P(HBco-HHx)/PEG blend. Vib. Spec. 60, 163-167. doi: 10.1016/j.vibspec.2012. 02.008

Kuo, S.-W., and Liu, W.-C. (2011). Miscibility enhancement through hydrogen bonding interaction of biodegradable poly(3-hydroxybutyrate) blending with poly(styrene-co-vinyl phenol) copolymer. J. Appl. Polymer Sci. 119, 300-310. doi: 10.1002/app.32528

Lai, H., and Wu, P. (2013). Hydration capabilities and structures of carbonyl and ether groups in poly(3-(2-methoxyethyl)-N-vinyl-2-pyrrolidone) film. Polym. Chem. 4, 3323-3332. doi: 10.1039/c3py00239j

Lee, C. O., Chae, B., Kim, S. B., Jung, Y. M., and Lee, S. W. (2012). Two-dimensional correlation analysis study of the photo-degradation of poly(ethylene terephthalate) film. Vib. Spec. 60, 142-145. doi: 10.1016/j.vibspec.2011.10.013

Lee, S. H., Chae, B., Kim, H.-C., Kim, S. B., Jung, Y. M., and Lee, S. W. (2010). Photoreaction and molecular reorientation studies of ultraviolet light irradiated azobenzene containing polymer films using twodimensional correlation infrared spectroscopy. J. Mol. Struct. 974, 35-39. doi: 10.1016/j.molstruc.2009.10.008

Li, X., Shen, Q., Zhang, D., Mei, X., Ran, W., Xu, Y., et al. (2013). Functional groups determine biochar properties ( $\mathrm{pH}$ and EC) as Studied by two-dimensional C NMR correlation spectroscopy. PLoS ONE 8:e65949. doi: 10.1371/journal.pone.0065949

Ma, L., Ahmed, Z., and Asher, S. A. (2011). Ultraviolet resonance raman study of side chain electrostatic control of poly-l-lysine conformation. J. Phys. Chem. B 115, 4251-4258. doi: 10.1021/jp2005343

Musto, P., Mensitieri, G., Lavorgna, M., Scarinzi, G., and Scherillo, G. (2011). Combining gravimetric and vibrational spectroscopy measurements to quantify first- and second-shell hydration layers in polyimides with different molecular architectures. J. Phys. Chem. B 116, 1209-1220. doi: 10.1021/jp20 56943

Noda, I. (1986). Two-dimensional infrared (2D IR) spectroscopy of synthetic and biopolymers. Bull. Am. Phys. Soc. 31:520.

Noda, I. (1993). Generalized two-dimensional correlation method applicable to infrared, raman, and other types of spectroscopy. Appl. Spec. 47, 1329-1336. doi: $10.1366 / 0003702934067694$

Noda, I. (2000). Progress in 2D correlation spectroscopy. AIP Conf. Proc. 503, 3-17. doi: $10.1063 / 1.1302843$

Noda, I. (2002). "General theory of two-dimensional (2D) analysis," in Handbook of Vibrational Spectroscopy, eds J. M. Chalmers, and P. R. Griffiths (Chichester: John Wiley \& Sons, Ltd), 2123-2134.

Noda, I. (2004). Advances in two-dimensional correlation spectroscopy. Vib. Spec. 36, 143-165. doi: 10.1016/j.vibspec.2003.12.016

Noda, I. (2006). Progress in two-dimensional (2D) correlation spectroscopy. J. Mol. Struct. 799, 2-15. doi: 10.1016/j.molstruc.2006.03.053 
Noda, I. (2007). Two-dimensional correlation analysis useful for spectroscopy, chromatography, and other analytical measurements. Anal. Sci. 23, 139-146. doi: 10.2116 /analsci.23.139

Noda, I. (2008). Recent advancement in the field of two-dimensional correlation spectroscopy. J. Mol. Struct. 883-884, 2-26. doi: 10.1016/j.molstruc.2007.11.038

Noda, I. (2009). "Chapter 13 - generalized two-dimensional correlation spectroscopy," in Frontiers of Molecular Spectroscopy, ed J. Laane (Amsterdam: Elsevier), 367-381.

Noda, I. (2010). Projection two-dimensional correlation analysis. J. Mol. Struct. 974, 116-126. doi: 10.1016/j.molstruc.2009.11.047

Noda, I. (2014a). Frontiers of two-dimensional correlation spectroscopy. part 1. New concepts and noteworthy developments. J. Mol. Struct. 1069, 3-22. doi: 10.1016/j.molstruc.2014.01.025

Noda, I. (2014b). Frontiers of two-dimensional correlation spectroscopy. Part 2. Perturbation methods, fields of applications, and types of analytical probes. J. Mol. Struct. 1069, 23-49. doi: 10.1016/j.molstruc.2014.01.016

Noda, I. (2014c). Two-dimensional correlation spectroscopy study of polystyrene. Macromol. Symp. 339, 17-23. doi: 10.1002/masy.201300129

Noda, I. (2014d). Vibrational spectroscopy in the development of surface hydrophilic elastomer latex (SHEL). Vib. Spec. 71, 70-75. doi: 10.1016/j.vibspec.2014.01.009

Noda, I., Dowrey, A. E., Marcoli, C., Story, G. M., and Ozaki, Y. (2000). Generalized two-dimensional correlation spectroscopy. Appl. Spec. 54, 236A-248A. doi: 10.1366/0003702001950454

Noda, I., Dowrey, A. E., and Marcott, C. (1993). Recent developments in two-dimensional infrared (2D IR) correlation spectroscopy. Appl. Spec. 47, 1317-1323. doi: 10.1366/0003702934067513

Noda, I., and Lindsey, S. B. (2010). "Plastics from Bacteria: natural functions and applications," in Microbiology Monographs, ed G. G.-Q. Chen (Berlin: Springer), 237-256.

Noda, I., and Ozaki, Y. (2004). Two-Dimensional Correlation Spectroscopy: Applications in Vibrational and Optical Spectroscopy. Chichester: John Wiley \& Sons Ltd. doi: 10.1002/0470012404

Oh, T.-J., Nam, J.-H., and Jung, Y. M. (2009). Molecular miscible blend of poly(2-cyano-1,4-phenyleneterephthalamide) and polyvinylpyrrolidone characterized by two-dimensional correlation FTIR and solid state 13C NMR spectroscopy. Vib. Spec. 51, 15-21. doi: 10.1016/j.vibspec.2008. 09.017

Ozaki, Y. (2002). "2D correlation spectroscopy in vibrational spectroscopy," in Handbook of Vibrational Spectroscopy, eds J. M. Chalmers, and P. R. Griffiths (John Wiley \& Sons, Ltd), 2135-2172.

Ozaki, Y., and Šašic, S. (2005). "Two-dimensional correlation spectroscopy of biological and polymeric materials," in Vibrational Spectroscopy of Biological and Polymeric Materials, eds V. G. Gregoriou and M. S. Braiman (New York, NY: CRC Press), 163-214.

Ozaki, Y., and Noda, I. (2006). "Two-dimensional vibrational correlation spectroscopy in biomedical sciences," in Encyclopedia of Analytical Chemistry, ed R. A. Meyers (John Wiley \& Sons, Ltd), 1-18.

Park, Y., Hashimoto, C., Hashimoto, T., Hirokawa, Y., Jung, Y. M., and Ozaki, Y. (2013). Reaction-induced self-assembly of gel structure: a new insight into chemical gelation process of $\mathrm{N}$-isopropylacrylamide as studied by twodimensional infrared correlation spectroscopy. Macromolecules 46, 3587-3602. doi: $10.1021 / \mathrm{ma} 400457 \mathrm{e}$

Park, Y., Kim, N. H., Choi, H. C., Lee, S. M., Hwang, H., Jeong, Y. U., et al. (2012). Two-dimensional hetero-spectral Raman/XAS correlation analysis of $\mathrm{Li}[\mathrm{Ni} 0.45 \mathrm{Co} 0.18 \mathrm{Mn} 0.25 \mathrm{Al} 0.12] \mathrm{O} 2$ cathode in the overcharged state. Vib. Spec. 60, 226-230. doi: 10.1016/j.vibspec.2012.03.004

Pazderka, T., and Kopecký Jr, V. (2012). Two-dimensional correlation analysis of Raman optical activity - Basic rules and data treatment. Vib. Spec. 60, 193-199. doi: 10.1016/j.vibspec.2011.10.002

Peng, H., Han, Y., Liu, T., Tjiu, W. C., and He, C. (2010). Morphology and thermal degradation behavior of highly exfoliated CoAl-layered double hydroxide/polycaprolactone nanocomposites prepared by simple solution intercalation. Thermochim. Acta 502, 1-7. doi: 10.1016/j.tca.2010. 01.009

Pi, F., Shinzawa, H., Czarnecki, M. A., Iwahashi, M., Suzuki, M., and Ozaki, Y. (2010). Self-assembling of oleic acid (cis-9-octadecenoic acid) and linoleic acid (cis-9, cis-12-octadecadienoic acid) in ethanol studied by time-dependent attenuated total reflectance (ATR) infrared (IR) and twodimensional (2D) correlation spectroscopy. J. Mol. Struct. 974, 40-45. doi: 10.1016/j.molstruc.2009.11.060

Popescu, M. C., and Vasile, C. (2011). Two-dimensional infrared correlation spectroscopic studies of polymer blends-Specific interactions in polyethylene adipate/cholesteryl palmitate blends. Spectrochim. Acta A Mol. Biomol. Spectrosc. 79, 45-50. doi: 10.1016/j.saa.2011.01.042

Popescu, M.-C., and Vasile, C. (2010). Melting behavior of polytetra hydrofuran/cholesteryl palmitate blends investigated by two-dimensional infrared correlation spectroscopy. Soft Mater. 8, 386-406. doi: $10.1080 / 1539445 X .2010 .525168$

Qu, Y., Huang, G., Wang, X., and Li, J. (2012). Study on the mechanism of the formation of polyhedral oligomeric silsesquioxanes by the $2 \mathrm{D}$ correlation infrared spectral. J. Appl. Polym. Sci. 125, 3658-3665. doi: 10.1002/ app.36489

Quaroni, L., Zlateva, T., and Normand, E. (2011). Detection of weak absorption changes from molecular events in time-resolved FT-IR spectromicroscopy measurements of single functional cells. Anal. Chem. 83, 7371-7380. doi $10.1021 / \mathrm{ac} 201318 \mathrm{z}$

Radice, S., Tommasini, M., and Castiglioni, C. (2010). Two dimensional correlation Raman spectroscopy of perfluoropolyethers: effect of peroxide groups. J. Mol. Struct. 974, 73-79. doi: 10.1016/j.molstruc.2009.11.063

Ryu, S. R., Bae, W. M., Hong, W. J., Ihn, K. J., and Jung, Y. M. (2012). Characterization of chain transfer reaction during radical polymerization of silver nanocomposite polyvinylpyrrolidone by using $2 \mathrm{D}$ hetero-spectral IR/NMR correlation spectroscopy. Vib. Spec. 60, 168-172. doi: 10.1016/j.vibspec.2011.12.009

Seo, H., Chae, B., Im, J. H., Jung, Y. M., and Lee, S. W. (2014). Imidization induced structural changes of 6FDA-ODA poly(amic acid) by two-dimensional (2D) infrared correlation spectroscopy. J. Mol. Struct. 1069, 196-199. doi: 10.1016/j.molstruc.2014.02.012

Shinzawa, H., Awa, K., Noda, I., and Ozaki, Y. (2013). Pressure-induced variation of cellulose tablet studied by two-dimensional (2D) near-infrared (NIR) correlation spectroscopy in conjunction with projection pretreatment. Vib. Spec. 65, 28-35. doi: 10.1016/j.vibspec.2012.11.009

Shinzawa, H., Awa, K., and Ozaki, Y. (2012). Compression effect on sustainedrelease and water absorption properties of cellulose tablets studied by heterospectral two-dimensional (2D) correlation analysis. Anal. Methods 4, 1530-1537. doi: 10.1039/clay05392b

Sikirzhytski, V., Topilina, N. I., Takor, G. A., Higashiya, S., Welch, J. T., Uversky, V. N., et al. (2012). Fibrillation mechanism of a model intrinsically disordered protein revealed by $2 \mathrm{D}$ correlation deep UV resonance raman spectroscopy. Biomacromolecules 13, 1503-1509. doi: 10.1021/bm300193f

Smirnova, D. S., Kornfield, J. A., and Lohse, D. J. (2011). Morphology development in model polyethylene via two-dimensional correlation analysis. Macromolecules 44, 6836-6848. doi: 10.1021/ma200774u

Su, Z., Jiang, Z., Huang, G., Li, L., and Wang, X. (2012). Mechanism of formation of partially crosslinked polyacrylamide complexes. J. Macromol. Sci. B 52, 22-35. doi: 10.1080/00222348.2012.687330

Tang, H., Sun, S., Wu, J., Wu, P., and Wan, X. (2010). Conformational changes in novel thermotropic liquid crystalline polymer without conventional mesogens: a raman spectroscopic investigation. Polymer 51, 5482-5489. doi: 10.1016/j.polymer.2010.09.036

Unger, M., Morita, S., Sato, H., Ozaki, Y., and Siesler, H. W. (2009). Variable-temperature fourier transform infrared spectroscopic investigations of poly(3-Hydroxyalkanoates) and perturbation-correlation moving-window two-dimensional correlation analysis. part ii: study of poly(\&epsi;-Caprolactone) homopolymer and a poly(3Hydroxybutyrate)\&ndash;Poly(\&epsi;-Caprolactone) Blend. Appl. Spec. 63, 1034-1040. doi: 10.1366/000370209789379240

Unger, M., Sato, H., Ozaki, Y., and Siesler, H. W. (2011). Crystallization behavior of poly(3-hydroxybutyrate) (PHB), Poly( $\varepsilon$-caprolactone) (PCL) and Their Blend (50:50?wt.\%) Studied by 2D FT-IR correlation spectroscopy. Macromol. Symp. 305, 90-100. doi: 10.1002/masy.201000135

Wang, L., Di, S., Wang, W., Chen, H., Yang, X., Gong, T., et al. (2014). Tunable temperature memory effect of photo-cross-linked star PCL-PEG networks. Macromolecules 47, 1828-1836. doi: 10.1021/ma4023229

Wang, X., Xiang, K., Nie, Y., Huang, G., Wu, J., and Su, Z. (2013). Intermediate state and weak intermolecular interactions of $\alpha$-trans-1,4-Polyisoprene during 
the gradual cooling crystallization process investigated by In situ FTIR and twodimensional infrared correlation spectroscopy. Macromol. Res. 21, 493-501. doi: 10.1007/s13233-013-1045-2

Wang, Z., and Wu, P. (2011). Spectral insights into gelation microdynamics of PNIPAM in an ionic liquid. J. Phys. Chem. B 115, 10604-10614. doi: 10.1021/jp205650h

Wu, Q.-Y., Chen, X.-N., Wan, L.-S., and Xu, Z.-K. (2012). Interactions between polyacrylonitrile and solvents: density functional theory study and twodimensional infrared correlation analysis. J. Phys. Chem. B 116, 8321-8330. doi: 10.1021/jp304167f

Wu, Y., Jiang, J.-H., and Ozaki, Y. (2002). A new possibility of generalized two-dimensional correlation spectroscopy:? hybrid two-dimensional correlation spectroscopy. J. Phys. Chem. A 106, 2422-2429. doi: 10.1021/ jp012140g

Wu, Y., Meersman, F., and Ozaki, Y. (2006). A novel application of hybrid two-dimensional correlation infrared spectroscopy:? exploration of the reversibility of the pressure- and temperature-induced phase separation of poly(N-isopropylacrylamide) and Poly(N-isopropylmethacrylamide) in aqueous solution. Macromolecules 39, 1182-1188. doi: 10.1021/ma 0521755

Zhang, M., Dang, Y.-Q., Liu, T.-Y., Li, H.-W., Wu, Y., Li, Q., et al. (2013). Pressure-induced fluorescence enhancement of the BSAprotected gold nanoclusters and the corresponding conformational changes of protein. J. Phys. Chem. C 117, 639-647. doi: 10.1021/ jp309175k

Zhang, M., Zhang, L., and Wu, Y. (2011). The pressure tolerance of different poly-l-lysine conformers in aqueous solution: infrared spectroscopy and two-dimensional correlation analysis. Vib. Spec. 57, 319-325. doi: 10.1016/j.vibspec.2011.09.007

Zheng, K., Liu, R., and Huang, Y. (2010). A two-dimensional IR correlation spectroscopic study of the conformational changes in syndiotactic polypropylene during crystallization. Polym. J. 42, 81-85. doi: 10.1038/pj.2009.304

Zhong, X., Liu, Y., Tang, X., Wu, Q., Li, L., and Yu, Y. (2012). Polyoxometalate cured epoxy resins with photochromic properties. Colloid Polym. Sci. 290, 1683-1693. doi: 10.1007/s00396-012-2688-9

Conflict of Interest Statement: The authors declare that the research was conducted in the absence of any commercial or financial relationships that could be construed as a potential conflict of interest.

Copyright (c) 2015 Park, Noda and Jung. This is an open-access article distributed under the terms of the Creative Commons Attribution License (CC BY). The use, distribution or reproduction in other forums is permitted, provided the original author(s) or licensor are credited and that the original publication in this journal is cited, in accordance with accepted academic practice. No use, distribution or reproduction is permitted which does not comply with these terms. 